\title{
Synthesis of sulfonamide-conjugated glycosyl-amino acid building blocks
}

Marie Lopez, Laurent F. Bornaghi, Sally-Ann Poulsen*

Eskitis Institute for Drug Discovery, Griffith University, Brisbane, Queensland 4111, Australia.

*Corresponding author. Telephone: +61 73735 7825; e-mail: $\underline{\text { s.poulsen@ @riffith.edu.au }}$ 


\begin{abstract}
The efficient synthesis of novel glycoconjugate amino acid building blocks wherein the amino acid and carbohydrate moieties are linked via a sulfonamide functional group is reported. The general reaction sequence consists of coupling a glycosyl thioacetate to an amino acid methyl ester followed by oxidation and deprotection of the carbohydrate moiety. We demonstrate the synthesis of derivatives from a range of amino acids, with reaction at either the $\alpha$-amino group of amino acid precursors or the sidechain $\varepsilon$-amino group of lysine precursors.
\end{abstract}

\title{
Key Words
}

glycopeptide; isostere; peptidomimetic; deacetylation; carbohydrate; chemical biology 


\section{Introduction}

The importance of carbohydrates and glycoconjugates in diverse biochemical processes has stimulated the development of neoglycoconjugates as fundamental tools for biological research. ${ }^{1,2}$ A central role for carbohydrate-protein conjugates in medicine is now well appreciated with glycoprotein based therapeutics representing more than one third of approved biopharmaceuticals, while glycopeptide antibiotics remain the frontline defence against a wide range of drug-resistant bacterial infections. ${ }^{3}$ Glycopeptides are formidable synthetic targets, with synthetic challenges far exceeding those associated with their oligopeptide counterparts. As well, native glycoproteins and glycopeptides comprise $O$-linked and $N$-linked glycosidic bonds and can lack the stability and bioavailability required in a therapeutic setting. ${ }^{2}$ Glycopeptide mimetics may confer advantages over their native analogues, including stability towards degrading enzymes, improved bioavailability and reduced clearance rates. In addition, a non-native link between carbohydrate and amino acid moieties may provide added opportunities for interactions with the biological target leading to enhanced affinity and/or improved specificity. The development of glycoconjugate amino acid building blocks with non-native structural features thus opens new possibilities for the synthesis of biologically relevant glycopeptide mimetics. ${ }^{1}$ Our group and others recently developed a synthesis for $S$-glycosyl sulfonamides and employed this new methodology to the synthesis of sulfonamide-bridged glycomimetics. ${ }^{4-6}$ In this contribution we further elaborate the scope of this chemistry to provide a simple, efficient and novel methodology for tethering sugar moieties to amino acids via a sulfonamide linker with potential for the application to a new class of glycopeptide mimetics.

\section{Results and discussion}

Our preliminary study was conducted using the simple amino acid glycine. The synthetic procedure towards carbohydrate-glycine glycoconjugates involved the reaction of $S$-acetyl thioglucose 1 with the $\alpha$-amino group of glycine methyl ester to give the glycoconjugate sulfenamide 2a, Scheme 1. Oxidation of $\mathbf{2 a}$ provided the sulfonamide glycoconjugate $\mathbf{2 b}$, wherein the carbohydrate and glycine methyl ester are linked by a sulfonamide group. Standard Zemplén conditions $^{7}$ for removal of the acetate protecting groups of the glycosyl moiety of $\mathbf{2 b}$ ( $0.05 \mathrm{M} \mathrm{NaOMe}$ in $\mathrm{MeOH}, \mathrm{pH} \sim 12$ ) were employed to provide the deprotected glycoconjugate as the methyl ester $\mathbf{2 c}$, or alternatively using $\mathrm{NaOH}$ in place of $\mathrm{NaOMe}$, to provide the deprotected 
glycoconjugate as the free acid $\mathbf{2 d}$. All reactions proceeded in high yield and purifications were straightforward, Table 1, entries 1 and 2.

Scheme 1. Synthesis of glycoconjugate glycine building blocks with a sulfonamide linker.

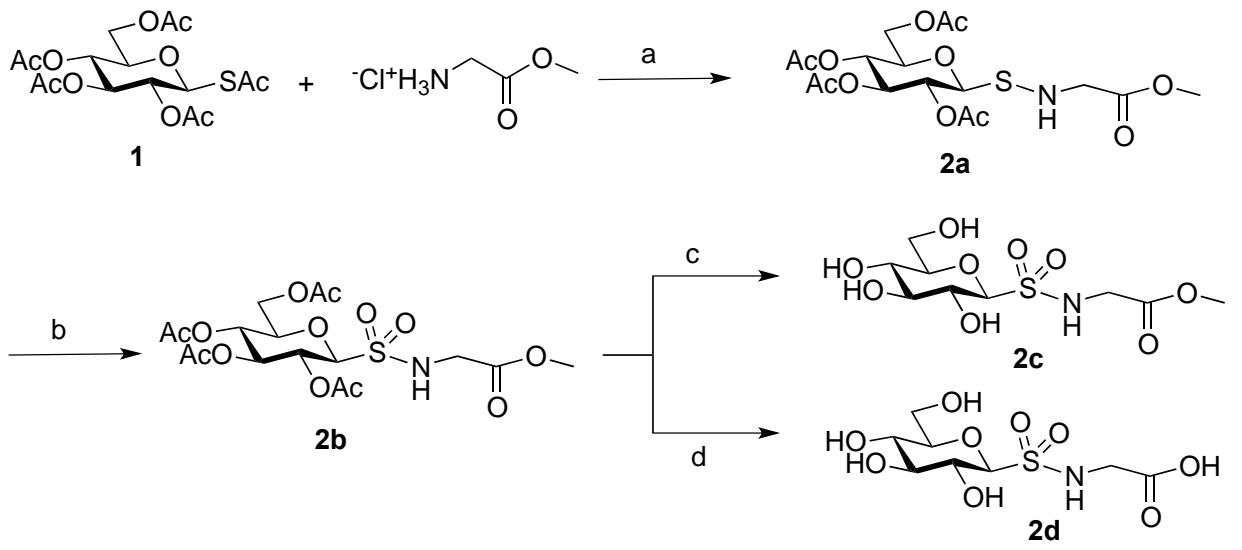

Reagents and conditions. (i) a) 2.5 equiv. $\mathrm{BrCH}\left(\mathrm{CO}_{2} \mathrm{Et}\right)_{2}, \mathrm{MeOH}, \mathrm{rt}, 20 \mathrm{~min}$; b) 3.0 equiv. glycine methyl ester, 3.0 equiv DIPEA, rt, $2 \mathrm{~h}, 96 \%$; (ii) 7.0 equiv. $m \mathrm{CPBA}, \mathrm{CH}_{2} \mathrm{Cl}_{2}, \mathrm{rt}, 1 \mathrm{~h}$, 87\%; (iii) $\mathrm{NaOMe}, \mathrm{MeOH}, \mathrm{rt}, 2 \mathrm{~h}, 94 \%$; (iv) $\mathrm{NaOH}, \mathrm{MeOH}, 0{ }^{\circ} \mathrm{C}$ to rt, $1 \mathrm{~h}$; then Amberlite IR120- $\mathrm{H}^{+}$, quantitative.

We next extended this preliminary study to a selection of amino acid derivatives that encompassed variable side chain properties including aliphatic (valine), aromatic (phenylglycine), polar/alcohol (serine) and conformationally restricted (proline), Table 1. These amino acids were available enantiomerically pure as their methyl ester. The chemistry to form glycoconjugates proceeded as in Scheme 1, however the sugar acetate deprotection step (Scheme 1, step iii) for both the phenylglycine and serine glycoconjugates $\mathbf{4 b}$ and $\mathbf{5 b}$ gave $\mathbf{4} \mathbf{c}^{\prime}$ and $\mathbf{5} \mathbf{c}^{\prime}$ in high yield but as a mixture of two diastereomers, Table 1 entries 4 and 6. To avoid this racemization alternate non-basic acetate deprotection reaction conditions were sought. We investigated the removal of the acetate protecting groups of $\mathbf{4 b}$ and $\mathbf{5 b}$ under mild acidic conditions $\left(8 \% \mathrm{HCl}\right.$ in methanol). ${ }^{8}$ The acetate groups were cleanly removed with retention of the amino acid $\alpha$-carbon stereochemistry to give glycoconjugates $\mathbf{4 c}(23 \%)$ and $\mathbf{5 c}(36 \%)$ as a single diastereomer, Table 1 entries 5 and 7. 
The biological activity of proteins and peptides may be enhanced by conjugation to carbohydrates. ${ }^{9}$ Synthetic strategies towards glycoconjugate amino acids may capitalize either on the inherent reactivity of the $\alpha$-amino, carboxylic acid or side chain functionality of native amino acids or on introduced chemoselective reactivity of a modified side chain in a non-native amino acid. $^{1,10-12}$ The glycoconjugate amino acids of our study were linked through reaction of the $\alpha$ amino group of the amino acid so it is expected that conjugation of a sugar to the free $\mathrm{N}$-terminus of a peptide or protein would be possible using this synthetic approach. In order to increase the generality of this method to allow site specific incorporation of a carbohydrate moiety into a peptide or protein it is necessary to apply this chemistry to the side chain of an amino acid. The sulfonamide linker has been previously employed as an amide bond isostere in the synthesis of peptidosulfonamide peptidomimetics. ${ }^{13}$ The $\varepsilon$-amino group of lysine is unique among native amino acids and lysine was selected as the candidate to evaluate side chain glycoconjugate formation through a sulfonamide linkage.

From L-lysine methyl ester dihydrochloride the disubstituted glycoconjugates 7a-c were synthesized, Table 1, entry 9. Reaction occurred without difficulty (monitored by TLC) at the $\alpha$ amino group while reaction of the sidechain $\varepsilon$-amino appeared relatively less favoured leading to a low yield of the disubstituted compound 7a (30\%). Similarly the reaction of $N^{\alpha}$-acetyl-L-lysine methyl ester or $N^{\alpha}$-fluorenylmethyloxycarbonyl-L-lysine with $\mathbf{1}$ also proceeded to form the sulfenamide-linked glycosyl amino acids 8a (24\%) and 9a (14\%) in low yields Table 2, entries 10 and 11. A reduced yield for the lysine sulfenamides 7a-9a compared to sulfenamides 2a-6a (yields $>80 \%$ ) identified a caveat to this methodology, which had so far proven high yielding. To avoid deacetylation of the sugar moiety of both reagent $\mathbf{1}$ and product sulfenamides it was necessary to maintain the $\mathrm{pH}$ value of the reaction (Scheme 1, step i) at $\sim \mathrm{pH} 9$. The $\mathrm{pK}_{\mathrm{a}}$ value of the side chain of the $\varepsilon$-amino group of lysine is however $\sim 10.5$, rendering it predominantly protonated under the reaction conditions. A balance between maximising the conversion to sulfenamide product while avoiding deacetylation of the carbohydrate moieties resulted in overall reduced yields for reaction of the side chain lysine $\varepsilon$-amino group compared to the $\alpha$-amino groups $\left(\mathrm{pK}_{\mathrm{a}}\right.$ values $\left.\sim 9-10\right)$. Despite this, the bis-sulfenamide 7a was formed in $30 \%$ yield, while the yield for 8a and 9a was $24 \%$ and $14 \%$, respectively, Table 1, entries 9-11. Given the ready 
availability and low cost of starting reagents this limitation does not significantly impact quantities of target compounds that may be synthesized.

Table 1. Glycoconjugate amino acid building blocks synthesized from $S$-acetyl thioglucose $\mathbf{1}$ and various amino acids.

\begin{tabular}{|c|c|c|c|c|}
\hline Entry & Amino acid & Product, Yield (\%) & Product, Yield (\%) & $\begin{array}{c}\text { Product, Yield } \\
(\%)\end{array}$ \\
\hline 1 & & 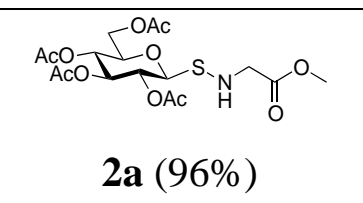 & 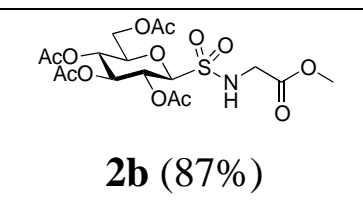 & 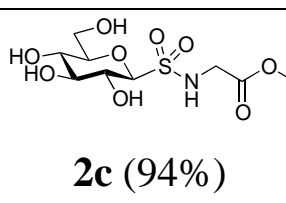 \\
\hline 2 & & $\mathbf{2 a}(96 \%)$ & $\begin{array}{c}\mathrm{OAC} \\
\mathbf{2 b}(87 \%)\end{array}$ & 2d (quantitative) \\
\hline 3 & & 3a $(90 \%)$ & 3b $(78 \%)$ & 3c $(93 \%)$ \\
\hline $4^{a}$ & & $\mathbf{4 a}(96 \%)$ & $\mathbf{4 b}(87 \%)$ & $\mathbf{4 c}^{\prime}(99 \%)$ \\
\hline $5^{b}$ & & $\mathbf{4 a}(96 \%)$ & $\mathbf{4 b}(87 \%)$ & 4c $(23 \%)$ \\
\hline $6^{a}$ & & $\mathbf{5 a}(81 \%)$ & $\mathbf{5 b}(57 \%)$ & $\mathbf{5 c}^{\prime}(99 \%)$ \\
\hline $7^{b}$ & & $\mathbf{5 a}(81 \%)$ & $\mathbf{5 b}(57 \%)$ & 5c $(36 \%)$ \\
\hline
\end{tabular}




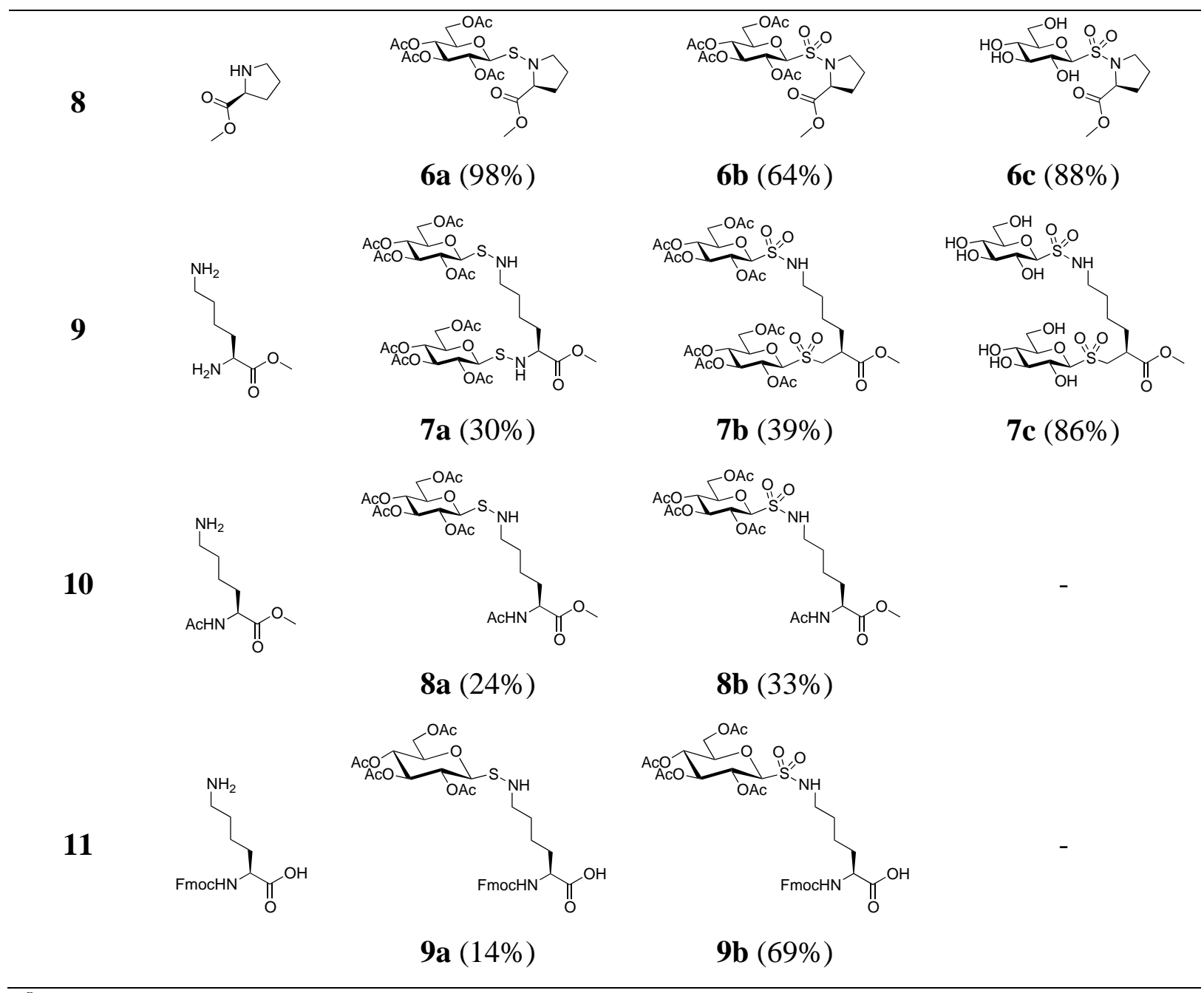

${ }^{a}$ Deprotection of per- $O$-acetylated glycoconjugates with basic conditions

${ }^{b}$ Deprotection of per- $O$-acetylated glycoconjugates with acidic conditions

\section{Experimental}

\subsection{General methods}

All starting materials and reagents, including per- $O$-acetylated glucopyranose, were purchased from commercial suppliers. 1-S-Acetyl-2,3,4,6-tetra- $O$-acetyl-1-thio- $\beta$-D-glucopyranose (1) was synthesized as described earlier. ${ }^{14}$ All reactions were monitored by TLC. TLC plates were visualized with UV light, ninhydrin stain $(1 \mathrm{~g}$ of ninhydrin in $100 \mathrm{~mL}$ of EtOH containing $3 \%$ $(\mathrm{v} / \mathrm{v})$ acetic acid) and/or orcinol stain (1 $\mathrm{g}$ of orcinol monohydrate in a mixture of EtOH: $\mathrm{H}_{2} \mathrm{O}: \mathrm{H}_{2} \mathrm{SO}_{4}$ 72.5:22.5:5 mL). Silica gel flash chromatography was performed using silica gel $60 \AA$ (230-400 mesh). ${ }^{1} \mathrm{H}$ NMR were acquired at $500 \mathrm{MHz}$ and ${ }^{13} \mathrm{C} \mathrm{NMR}$ at $125 \mathrm{MHz}$ at 30 ${ }^{\circ} \mathrm{C}$. For ${ }^{1} \mathrm{H}$ and ${ }^{13} \mathrm{C}$ NMR acquired in $\mathrm{CDCl}_{3}$ chemical shifts $(\delta)$ are reported in ppm relative to 
the solvent residual peak: proton $(\delta 7.27 \mathrm{ppm})$ and carbon $(\delta 77.2 \mathrm{ppm})$. Chemical shifts for ${ }^{1} \mathrm{H}$ and ${ }^{13} \mathrm{C}$ NMR acquired in DMSO- $d_{6}$ are reported in ppm relative to residual solvent proton $(\delta$ $2.50 \mathrm{ppm})$ and carbon $(\delta 39.5 \mathrm{ppm})$ signals, respectively. Assignments for ${ }^{1} \mathrm{H}$ NMR were confirmed by ${ }^{1} \mathrm{H}-{ }^{1} \mathrm{H}$ gCOSY, while assignments for ${ }^{13} \mathrm{C}$ NMR were confirmed by ${ }^{1} \mathrm{H}-{ }^{13} \mathrm{C}$ HSQC. Multiplicity is indicated as follows: s (singlet); d (doublet); t (triplet); m (multiplet); dd (doublet of doublet); ddd (doublet of doublet of doublet); br (broad). Coupling constants are reported in Hertz $(\mathrm{Hz})$. Melting points are uncorrected. High and low resolution electrospray ionization mass spectra were acquired using electrospray as the ionization technique in positive ion and/or negative ion modes as stated. All MS analysis samples were prepared as solutions in $\mathrm{MeOH}$. Optical rotations were measured at $25{ }^{\circ} \mathrm{C}$ with $\mathrm{Na}-589 \mathrm{~nm}$ wave length and a $100 \mathrm{~mm}$ cell and reported as an average of ten measurements. Purity of all compounds was $\geq 95 \%$ by NMR. Glycoconjugates are named in accordance with the recommendations of the IUPAC-IUBMB Joint Commission on Biochemical Nomenclature: "Nomenclature of Carbohydrates (Recommendations 1996)" (http://www.chem.qmul.ac.uk/iupac/2carb/).

\subsection{Synthesis methods}

\subsubsection{General procedure 1 - Synthesis of sulfenamide linked glycoconjugates} 1-S-Acetyl-2,3,4,6-tetra- $O$-acetyl-1-thio- $\beta$-D-glucopyranose (1) (1 equiv.) was dissolved in anhydrous $\mathrm{MeOH}$ under nitrogen. Diethyl bromomalonate (2.5 equiv.) was added and the reaction stirred at $\mathrm{rt}$ under nitrogen for $20 \mathrm{~min}$, then the amino acid methyl ester derivative (3.0 equiv.) and DIPEA (3.0 equiv.) added. The reaction was stirred at $\mathrm{rt}$ under nitrogen until complete as evidenced by TLC, typically $2 \mathrm{~h}$. The $\mathrm{MeOH}$ was removed and the residue dissolved in $\mathrm{CH}_{2} \mathrm{Cl}_{2}$ and washed with brine $(\times 3)$. Each aqueous fraction was back extracted with $\mathrm{CH}_{2} \mathrm{Cl}_{2}$ $(\times 2)$. The combined organic fractions were dried over $\mathrm{MgSO}_{4}$, filtered and the solvent removed to give the product sulfenamides $\mathbf{2 a - 6 a , 8 a - 9 a . ~}$

\subsubsection{General procedure 2 - Oxidation of sulfenamide linked glycoconjugates}

The sulfenamide derivative (1.0 equiv.) was dissolved in $\mathrm{CH}_{2} \mathrm{Cl}_{2}$. meta-Chloroperoxybenzoic acid (7.0 equiv.) in $\mathrm{CH}_{2} \mathrm{Cl}_{2}$, was added dropwise over $\sim 20$ min. The reaction was maintained at $\mathrm{rt}$ until full disappearance of the starting material, as evidenced by TLC, typically $1 \mathrm{~h}$. The reaction

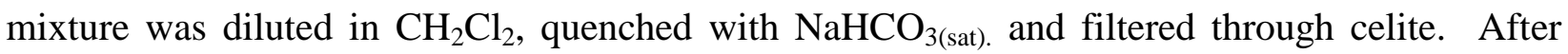


extraction with $\mathrm{CH}_{2} \mathrm{Cl}_{2}$, the organic fraction was washed with $\mathrm{NaHCO}_{3 \text { (sat). }}(\times 1)$ and brine $(\times 1)$. The aqueous fractions were back extracted with $\mathrm{CH}_{2} \mathrm{Cl}_{2}(\times 2)$. The organic fractions were then

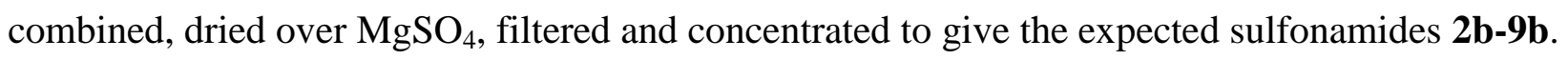

\subsubsection{General procedure 3 - Deprotection of per- $O$-acetylated glycoconjugates with basic conditions}

Fully deprotected glycoconjugates were prepared by treating a solution of the per- $O$-acetylated sulphonamide glycoconjugates $\mathbf{2 b - 7 b}$ (1.0 equiv.) in anhydrous $\mathrm{MeOH}$ at $0{ }^{\circ} \mathrm{C}$ with methanolic sodium methoxide ( $0.05 \mathrm{M}$ final concentration), $\mathrm{pH} 12$. The reaction was warmed to $\mathrm{rt}$ and left to stir until full deprotection as evident by TLC $(\sim 1 \mathrm{~h})$. The solution was neutralized with Amberlite IR-120 $\left[\mathrm{H}^{+}\right]$, filtered and the resin washed several times with $\mathrm{MeOH}$. The solvent was evaporated under reduced pressure, the residue redissolved in water and lyophilized to afford fully deprotected glycoconjugates $\mathbf{2 c}, \mathbf{3 c}, \mathbf{4} \mathbf{c}^{\prime}, \mathbf{5} \mathbf{c}^{\prime}, \mathbf{6 c}, \mathbf{7 c}$.

\subsubsection{General procedure 4 - Deprotection of per- $O$-acetylated glycoconjugates with acidic conditions}

A solution of the per- $O$-acetylated glycoconjugate $(\mathbf{4 b}$ or $\mathbf{5 b}$ ) was stirred overnight in $8 \% \mathrm{HCl}$ in $\mathrm{MeOH}$. The reaction mixture was concentrated in the presence of silica gel and the crude product purified by flash chromatography to afford the fully deprotected and stereochemically pure sulfonamide glycoconjugates $\mathbf{4 c}$ or $\mathbf{5 c}$, respectively.

\subsection{Methyl $N$-[(2,3,4,6-tetra- $O$-acetyl- $\beta$-D-glucosyl)thio]-glycinate (2a)}

Glucoside 2a was prepared from 1 and L-glycine methyl ester hydrochloride according to the general procedure 1. After purification by flash chromatography (1:2 EtOAc/hexane) the expected compound was obtained as an orange oil (96\%): $[\alpha]_{\mathrm{D}}{ }^{25}-102\left(c 1.0, \mathrm{CHCl}_{3}\right) ; \mathrm{R}_{f} 0.41(2: 3$ EtOAc/hexane); ${ }^{1} \mathrm{H}$ NMR (500 MHz, DMSO-d $): \delta 5.33$ (t, $\left.1 \mathrm{H}, J 9.5 \mathrm{~Hz}, \mathrm{H}-3\right), 5.08$ (t, $1 \mathrm{H}, J 9.5$ Hz, H-2), 4.86 (t, 1H, J 9.5 Hz, H-4), 4.62 (d, 1H, J 10.0 Hz, H-1), 4.09-4.01 (m, 3H, H-6a, H-6b, $\mathrm{NH}), 3.97$ (ddd, 1H, J 2.5, 5.5, 7.0 Hz, H-5), 3.65 (d, 2H, J 6.5 Hz, NHCH 2$), 3.64$ (s, 3H, $\mathrm{OCH}_{3}$ ), $2.02,2.01,1.99,1.96\left(4 \times \mathrm{s}, 12 \mathrm{H}, \mathrm{OCOCH}_{3}\right) ;{ }^{13} \mathrm{C} \mathrm{NMR}\left(125 \mathrm{MHz}, \mathrm{DMSO}-d_{6}\right): \delta 172.2$ $\left(\mathrm{CO}_{2} \mathrm{CH}_{3}\right), 170.0,169.5,169.4,169.3\left(4 \times \mathrm{OCOCH}_{3}\right), 87.4(\mathrm{C}-1), 74.3(\mathrm{C}-5), 73.1(\mathrm{C}-3), 68.1$ (C-4), 67.3 (C-2), 62.1 (C-6), $54.6\left(\mathrm{NHCH}_{2}\right), 51.5\left(\mathrm{CO}_{2} \mathrm{CH}_{3}\right), 20.5,20.5,20.3,20.2(4 \times$ 
$\left.\mathrm{OCOCH}_{3}\right)$; LRMS $\left(\mathrm{ESI}^{+}\right): \mathrm{m} / z 474[\mathrm{M}+\mathrm{Na}]^{+}$; HRMS: Calcd for $\mathrm{C}_{17} \mathrm{H}_{25} \mathrm{NO}_{11} \mathrm{SNa}[\mathrm{M}+\mathrm{Na}]^{+}$ 474.1041, Found 474.1053.

\subsection{Methyl $N$-[(2,3,4,6-tetra- $O$-acetyl- $\beta$-D-glucosyl)thio]-L-valinate (3a)}

Glucoside 3a was prepared from $\mathbf{1}$ and L-valine methyl ester hydrochloride according to the general procedure 1. After purification by flash chromatography (1:2 EtOAc/hexane) the expected compound was obtained as a slightly yellow solid (90\%): mp $90-93{ }^{\circ} \mathrm{C} ;[\alpha]_{\mathrm{D}}{ }^{25}-138(c$ $\left.1.0, \mathrm{CHCl}_{3}\right) ; \mathrm{R}_{f} 0.41$ (2:3 EtOAc/hexane); ${ }^{1} \mathrm{H}$ NMR (500 MHz, DMSO- $\left.d_{6}\right): \delta 5.33$ (t, $1 \mathrm{H}, J 9.5$ Hz, H-3), 5.06 (t, 1H, J $9.5 \mathrm{~Hz}, \mathrm{H}-2$ ), 4.84 (t, 1H, J $9.5 \mathrm{~Hz}, \mathrm{H}-4$ ), 4.61 (d, 1H, J 10.5 Hz, H-1), 4.16 (dd, 1H, J 4.5, 12.0 Hz, H-6a), 3.99 (dd, 1H, J 2.5, 12.5 Hz, H-6b), 3.95 (m, 1H, H-5), 3.79 (d, $1 \mathrm{H}, J 9.5 \mathrm{~Hz}, \mathrm{NH}$ ), 3.64 (s, 3H, $\mathrm{OCH}_{3}$ ), 3.28 (br d, 1H, J $9.5 \mathrm{~Hz}, \mathrm{NHCH}$ ), 2.00 (s, 6H, $2 \times$ $\left.\mathrm{OCOCH}_{3}\right), 1.98\left(\mathrm{~s}, 3 \mathrm{H}, \mathrm{OCOCH}_{3}\right), 1.95\left(\mathrm{~s}, 3 \mathrm{H}, \mathrm{OCOCH}_{3}\right), 1.93\left(\mathrm{~m}, 1 \mathrm{H}, \mathrm{CH}_{3} \mathrm{CHCH}_{3}\right), 0.93$ (d, $3 \mathrm{H}, J 7.0 \mathrm{~Hz}, \mathrm{CH}_{3} \mathrm{CHCH}_{3}$ ), 0.88 (d, 3H, J 7.0 Hz, $\left.\mathrm{CH}_{3} \mathrm{CHCH}_{3}\right) ;{ }^{13} \mathrm{C}$ NMR (125 MHz, DMSO$\left.d_{6}\right): \delta 174.4\left(\mathrm{CO}_{2} \mathrm{CH}_{3}\right), 170.3,169.9,169.6,169.5\left(4 \times \mathrm{OCOCH}_{3}\right), 87.2(\mathrm{C}-1), 74.4(\mathrm{C}-5), 73.4$ (C-3), $72.5(\mathrm{NHCH}), 68.0(\mathrm{C}-4), 67.3(\mathrm{C}-2), 61.9(\mathrm{C}-6), 51.8\left(\mathrm{CO}_{2} \mathrm{CH}_{3}\right), 31.5\left(\mathrm{CH}_{3} \mathrm{CHCH}_{3}\right)$, 20.7, 20.6, $20.5(2 \mathrm{C})\left(4 \times \mathrm{OCOCH}_{3}\right), 19.5,18.2\left(\mathrm{CH}_{3} \mathrm{CHCH}_{3}\right)$; LRMS $\left(\mathrm{ESI}^{+}\right): m / z, 494[\mathrm{M}+\mathrm{H}]^{+}$, $516[\mathrm{M}+\mathrm{Na}]^{+}$; HRMS: Calcd for $\mathrm{C}_{20} \mathrm{H}_{31} \mathrm{NO}_{11} \mathrm{SNa}[\mathrm{M}+\mathrm{Na}]^{+}$516.1510, Found 516.1511.

\subsection{Methyl $N$-[(2,3,4,6-tetra- $O$-acetyl- $\beta$-D-glucosyl)thio]- $R$-phenylglycinate (4a)}

Glucoside $\mathbf{4 a}$ was prepared from $\mathbf{1}$ and $R$-phenylglycine methyl ester hydrochloride according to the general procedure 1. After purification by flash chromatography (1:2 EtOAc/hexane) the expected compound was obtained as a yellow oil (96\%): $[\alpha]_{\mathrm{D}}^{25}-135$ (c 1.0, $\left.\mathrm{CHCl}_{3}\right) ; \mathrm{R}_{f} 0.58(1: 1$ EtOAc/hexane); ${ }^{1} \mathrm{H}$ NMR (500 MHz, DMSO- $\left.d_{6}\right): \delta 7.36-7.29$ (m, 5H, $\mathrm{H}_{\text {arom. }}$ ), 5.35 (t, $1 \mathrm{H}, J 9.5$ Hz, H-3), 5.13 (t, 1H, J 9.5 Hz, H-2), 4.90 (t, 1H, J 9.5 Hz, H-4), 4.73 (d, 1H, J 7.0 Hz, NHCH), 4.64 (d, 1H, J 7.0 Hz, NH), 4.62 (d, 1H, J 10.0 Hz, H-1), 4.09-4.05 (m, 2H, H-6a, H-6b), 3.98 (ddd, $1 \mathrm{H}, J$ 2.5, 5.5, $10.0 \mathrm{~Hz}, \mathrm{H}-5), 3.63\left(\mathrm{~s}, 3 \mathrm{H}, \mathrm{OCH}_{3}\right), 2.03,1.99,1.98,1.97(4 \times \mathrm{s}, 12 \mathrm{H}$, $\left.\mathrm{OCOCH}_{3}\right) ;{ }^{13} \mathrm{C}$ NMR $\left(125 \mathrm{MHz}\right.$, DMSO- $\left.d_{6}\right): \delta 172.5\left(\mathrm{CO}_{2} \mathrm{CH}_{3}\right), 170.0,169.7,169.5,169.3(4 \times$ $\left.\mathrm{OCOCH}_{3}\right), 138.0\left(\mathrm{C}_{\text {arom. }}\right), 129.4,129.4,128.0,127.6,127.6\left(5 \times \mathrm{CH}_{\text {arom. }}\right), 87.3(\mathrm{C}-1), 74.4(\mathrm{C}-5)$, 72.9 (C-3), $68.7(\mathrm{NHCH}), 68.1(\mathrm{C}-4), 67.4(\mathrm{C}-2), 62.2(\mathrm{C}-6), 52.1\left(\mathrm{CO}_{2} \mathrm{CH}_{3}\right), 20.7,20.4,20.3$, $20.2\left(4 \times \mathrm{OCOCH}_{3}\right) ; \mathrm{LRMS}\left(\mathrm{ESI}^{+}\right): \mathrm{m} / z 528[\mathrm{M}+\mathrm{H}]^{+}$; HRMS: Calcd for $\mathrm{C}_{23} \mathrm{H}_{30} \mathrm{NO}_{11} \mathrm{SNa}[\mathrm{M}+$ $\mathrm{Na}]^{+}$528.1534, Found 528.1534. 


\subsection{Methyl $N$-[(2,3,4,6-tetra- $O$-acetyl- $\beta$-D-glucosyl)thio]-L-serinate (5a)}

Glucoside 5a was prepared from 1 and L-serine methyl ester hydrochloride according to the general procedure 1. After purification by flash chromatography (3:2 EtOAc/hexane) the expected compound was obtained as an orange oil $(81 \%)$ : $[\alpha]_{\mathrm{D}}^{25}-143\left(c 1.0, \mathrm{CHCl}_{3}\right) ; \mathrm{R}_{f} 0.22(1: 1$ EtOAc/hexane); ${ }^{1} \mathrm{H}$ NMR (500 MHz, DMSO- $\left.d_{6}\right): \delta 5.33$ (t, $\left.1 \mathrm{H}, J 9.5 \mathrm{~Hz}, \mathrm{H}-3\right), 5.01$ (t, $1 \mathrm{H}, J 9.5$ Hz, H-2), 4.90 (t, 1H, J 5.0 Hz, CH ${ }_{2} \mathrm{OH}$ ), 4.89 (t, 1H, J 9.5 Hz, H-4), 4.60 (d, 1H, J 9.5 Hz, H-1), 4.10 (dd, 1H, J 4.5, $12.5 \mathrm{~Hz}, \mathrm{H}-6 \mathrm{a}$ ), 4.05 (dd, 1H, J 2.5, $12.5 \mathrm{~Hz}, \mathrm{H}-6 \mathrm{~b}$ ), 3.96 (ddd, 1H, J 2.5, 4.5, $10.0 \mathrm{~Hz}, \mathrm{H}-5), 3.86$ (d, $1 \mathrm{H}, J 8.0 \mathrm{~Hz}, \mathrm{NH}$ ), 3.66 (dd, 2H, J 5.0, $10.0 \mathrm{~Hz}, \mathrm{CH}_{2} \mathrm{OH}$ ), 3.64 (s, 3H, $\left.\mathrm{CO}_{2} \mathrm{CH}_{3}\right), 3.55(\mathrm{~m}, 1 \mathrm{H}, \mathrm{NHCH}), 2.01\left(\mathrm{~s}, 6 \mathrm{H}, 2 \times \mathrm{OCOCH}_{3}\right), 1.98\left(\mathrm{~s}, 3 \mathrm{H}, \mathrm{OCOCH}_{3}\right), 1.95(\mathrm{~s}, 3 \mathrm{H}$, $\left.\mathrm{OCOCH}_{3}\right) ;{ }^{13} \mathrm{C}$ NMR $\left(125 \mathrm{MHz}, \mathrm{DMSO}-d_{6}\right): \delta 172.5\left(\mathrm{CO}_{2} \mathrm{CH}_{3}\right), 170.0,169.5,169.3,169.2(4 \times$ $\mathrm{OCOCH}_{3}$ ), 87.1 (C-1), 74.1 (C-5), 73.0 (C-3), 67.9 (C-4), $67.8(\mathrm{NHCH}), 67.3(\mathrm{C}-2), 62.3$ $\left(\mathrm{CH}_{2} \mathrm{OH}\right), 61.8(\mathrm{C}-6), 51.6\left(\mathrm{CO}_{2} \mathrm{CH}_{3}\right), 20.5,20.4,20.3,20.2\left(4 \times \mathrm{OCOCH}_{3}\right)$; LRMS $\left(\mathrm{ESI}^{+}\right): \mathrm{m} / \mathrm{z}$ $482[\mathrm{M}+\mathrm{H}]^{+}, 504[\mathrm{M}+\mathrm{Na}]^{+}$; HRMS: Calcd for $\mathrm{C}_{18} \mathrm{H}_{28} \mathrm{~N}_{1} \mathrm{O}_{12} \mathrm{~S} 482.1327$, Found 482.1303.

\subsection{Methyl $N$-[(2,3,4,6-tetra- $O$-acetyl-p-D-glucosyl)thio]-L-prolinate (6a)}

Glucoside 6a was prepared from 1 and L-proline methyl ester hydrochloride according to the general procedure 1. After purification by flash chromatography (2:3 EtOAc/hexane) expected compound was obtained as an off white solid (98\%): mp 89-90 ${ }^{\circ} \mathrm{C} ;[\alpha]_{\mathrm{D}}{ }^{25}-101\left(c 1.0, \mathrm{CHCl}_{3}\right) ; \mathrm{R}_{f}$ 0.53 (1:1 EtOAc/hexane); ${ }^{1} \mathrm{H}$ NMR (500 MHz, DMSO- $\left.d_{6}\right): \delta 5.31$ (t, $\left.1 \mathrm{H}, J 9.5 \mathrm{~Hz}, \mathrm{H}-3\right), 5.01$ (d, 1H, $J 10.5 \mathrm{~Hz}, \mathrm{H}-1), 4.88$ (t, 1H, J 9.5 Hz, H-4), 4.77 (t, 1H, J 9.5 Hz, H-2), 4.12 (dd, 1H, J 5.0, 12.5 Hz, H-6a), 4.02 (dd, 1H, J 2.5, 13.0 Hz, H-6b), 3.99 (m, 1H, H-5), 3.84 (dd, 1H, J 4.0, 9.0 $\mathrm{Hz}, \alpha-\mathrm{CH}), 3.63\left(\mathrm{~s}, 3 \mathrm{H}, \mathrm{OCH}_{3}\right), 3.19\left(\mathrm{~m}, 2 \mathrm{H}, \delta-\mathrm{CH}_{2}\right), 2.19(\mathrm{~m}, 1 \mathrm{H}, \beta-\mathrm{CH}), 1.82-1.78(\mathrm{~m}, 3 \mathrm{H}$, $\left.\beta-\mathrm{CH} H, \delta-\mathrm{CH}_{2}\right), 2.01,1.99,1.98,1.94\left(4 \times \mathrm{s}, 12 \mathrm{H}, \mathrm{OCOCH}_{3}\right) ;{ }^{13} \mathrm{C}$ NMR $\left(125 \mathrm{MHz}, \mathrm{DMSO}-d_{6}\right)$ : $\delta 172.5\left(\mathrm{CO}_{2} \mathrm{CH}_{3}\right), 170.0,169.5,169.3,169.2\left(4 \times \mathrm{OCOCH}_{3}\right), 85.8(\mathrm{C}-1), 74.2(\mathrm{C}-5), 73.1(\mathrm{C}-3)$, $68.0(\mathrm{C}-4), 67.8(\mathrm{C}-2), 66.9(\alpha-\mathrm{CH}), 62.0(\mathrm{C}-6), 56.6\left(\delta-\mathrm{CH}_{2}\right), 51.6\left(\mathrm{CO}_{2} \mathrm{CH}_{3}\right), 30.4\left(\beta-\mathrm{CH}_{2}\right)$, $24.4\left(\gamma-\mathrm{CH}_{2}\right), 20.4,20.3,20.3,20.2\left(4 \times \mathrm{OCOCH}_{3}\right) ; \mathrm{LRMS}\left(\mathrm{ESI}^{+}\right): \mathrm{m} / z 492[\mathrm{M}+\mathrm{H}]^{+}, 514[\mathrm{M}+$ $\mathrm{Na}^{+}$; HRMS: Calcd for $\mathrm{C}_{20} \mathrm{H}_{29} \mathrm{NO}_{11} \mathrm{SNa}[\mathrm{M}+\mathrm{Na}]^{+}$514.1354, Found 514.1336.

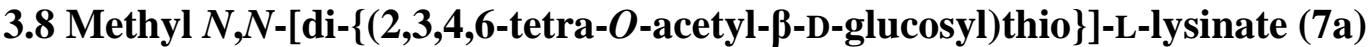


A solution of compound 1 (347 mg, $0.85 \mathrm{mmol}, 1.0$ equiv.) in anhydrous $\mathrm{MeOH}$ (10 mL) was stirred for $30 \mathrm{~min}$ under argon in presence of diethyl bromomalonate $(0.37 \mathrm{~mL}, 2.17 \mathrm{mmol}, 2.6$ equiv.). A solution of L-lysine methyl ester hydrochloride (605 mg, $2.60 \mathrm{mmol}, 3.1 \mathrm{equiv}$.) in anhydrous $\mathrm{MeOH}(20 \mathrm{~mL})$ and DIPEA (0.9 mL, $5.17 \mathrm{mmol}, 6.0$ equiv.) were added and the mixture was stirred under argon at $\mathrm{rt}$ for $2 \mathrm{~h}$. The solvent was evaporated and the residue was dissolved in $\mathrm{CH}_{2} \mathrm{Cl}_{2}$ and washed with brine $(\times 2)$. After back extraction of each aqueous fraction with $\mathrm{CH}_{2} \mathrm{Cl}_{2}(\times 2)$ the organic fractions were combined, dried over $\mathrm{MgSO}_{4}$, filtered and concentrated to afford a monosubstituted derivative used for the next step as a crude mixture. The same procedure as above was repeated to afford the second substitution. Purification by flash chromatography (1:1 EtOAc/hexane) afforded the title compound 7a as a yellow oil (30\%): $[\alpha]_{\mathrm{D}}{ }^{25}+106\left(c\right.$ 1.0, $\left.\mathrm{CHCl}_{3}\right) ; \mathrm{R}_{f} 0.64$ (2:1 EtOAc/hexane); ${ }^{1} \mathrm{H}$ NMR (500 MHz, DMSO- $\left.d_{6}\right): \delta 5.32$ $\left(2 \times \mathrm{t}, 2 \mathrm{H}, J 9.0 \mathrm{~Hz}, \mathrm{H}-3, \mathrm{H}-3^{\prime}\right), 4.96$ (t, 1H, J $9.5 \mathrm{~Hz}, \mathrm{H}-2$ or H-2'), 4.90-4.83 (m, 3H, H-2 or H2’, H-4, H-4’), 4.69, 4.63 (2 × d, 2H, J 10.0 Hz, H-1, H-1' ), 4.14-4.10 (m, 2H, H-6a, H-6a'), 4.05-4.02 (m, 2H, NH, H-6b or H-6b'), 4.00 (dd, 1H, J 12.5, 2.0 Hz, H-6b or H-6b'), 3.95 (2 × ddd, 2H, J 10.0, 4.5, 2.0 Hz, H-5, H-5'), 3.74 (t, 1H, J 5.5 Hz, ع-NH), 3.63 (s, 3H, OCH ) $_{3} 3.45$ $(\mathrm{td}, 1 \mathrm{H}, J$ 8.0, $6.0 \mathrm{~Hz}, \alpha-\mathrm{CH}), 2.82\left(\mathrm{~m}, 2 \mathrm{H}, \varepsilon-\mathrm{CH}_{2}\right), 2.02\left(\mathrm{~s}, 3 \mathrm{H}, \mathrm{OCOCH}_{3}\right), 2.01(\mathrm{~s}, 6 \mathrm{H}, 2 \times$ $\left.\mathrm{OCOCH}_{3}\right), 2.00\left(\mathrm{~s}, 3 \mathrm{H}, \mathrm{OCOCH}_{3}\right), 1.98\left(\mathrm{~s}, 6 \mathrm{H}, 2 \times \mathrm{OCOCH}_{3}\right), 1.94\left(\mathrm{~s}, 6 \mathrm{H}, 2 \times \mathrm{OCOCH}_{3}\right), 1.58$ $\left(\mathrm{m}, 2 \mathrm{H}, \beta-\mathrm{CH}_{2}\right), 1.42\left(\mathrm{~m}, 2 \mathrm{H}, \delta-\mathrm{CH}_{2}\right), 1.34\left(\mathrm{~m}, 2 \mathrm{H}, \gamma-\mathrm{CH}_{2}\right) ;{ }^{13} \mathrm{C}$ NMR (125 MHz, DMSO- $\left.d_{6}\right): \delta$ $174.3\left(\mathrm{CO}_{2} \mathrm{CH}_{3}\right), 169.9,169.9,169.5,169.2,169.2,169.2,169.1,168.4\left(8 \times \mathrm{OCOCH}_{3}\right), 87.6$, 87.4 (C-1, C-1') , 74.2, 74.2 (C-5, C-5’), 73.2, 73.2 (C-3, C-3’), 68.1, 68.0 (C-4, C-4'), 67.6, 67.4 $\left(\mathrm{C}-2, \mathrm{C}^{\prime} 2^{\prime}\right), 65.5(\alpha-\mathrm{CH}), 61.9,61.9\left(\mathrm{C}-6, \mathrm{C}-6^{\prime}\right), 52.6\left(\varepsilon-\mathrm{CH}_{2}\right), 51.6\left(\mathrm{CO}_{2} \mathrm{CH}_{3}\right), 32.2\left(\beta-\mathrm{CH}_{2}\right)$, $29.3\left(\delta-\mathrm{CH}_{2}\right), 22.5\left(\gamma-\mathrm{CH}_{2}\right), 20.4,20.4,20.3(\times 6)\left(8 \times \mathrm{OCOCH}_{3}\right) ; \mathrm{LRMS}\left(\mathrm{ESI}^{+}\right): \mathrm{m} / z 885[\mathrm{M}+$ $\mathrm{H}]^{+}$; HRMS: Calcd for $\mathrm{C}_{35} \mathrm{H}_{53} \mathrm{~N}_{2} \mathrm{O}_{20} \mathrm{~S}_{2}$ 885.2628, Found 885.2669.

\section{9 $N^{\alpha}$-Fluorenylmethyloxycarbonyl- $N^{\varepsilon}$-[(2,3,4,6-tetra- $O$-acetyl- $\beta$-D-glucosyl)thio]-L-lysine} (8a)

The title compound 8a was prepared from $N^{\alpha}$-fluorenylmethyloxycarbonyl-L-lysine according to the general procedure 1. Purification by flash chromatography (4:1 EtOAc/petroleum spirit + $\mathrm{AcOH} 1.5 \% \mathrm{v} / \mathrm{v})$ afforded the title compound 8a as a colorless oil $(14 \%):[\alpha]_{\mathrm{D}}{ }^{25}-94(c$ 1.0, $\left.\mathrm{CHCl}_{3}\right) ; \mathrm{R}_{f} 0.24(4: 1 \mathrm{EtOAc/hexane}+1.5 \%$ acetic acid $) ;{ }^{1} \mathrm{H}$ NMR $\left(500 \mathrm{MHz}, \mathrm{DMSO}-d_{6}\right): \delta 7.89$ 
(d, 2H, J 7.5 Hz, $\left.\mathrm{H}_{\text {arom. }}\right), 7.72\left(\mathrm{~d}, 2 \mathrm{H}, J 7.5 \mathrm{~Hz}, \mathrm{H}_{\text {arom. }}\right), 7.42\left(\mathrm{t}, 2 \mathrm{H}, J 7.5 \mathrm{~Hz}, \mathrm{H}_{\text {arom. }}\right), 7.33$ (t, 2H, $J$ $7.5 \mathrm{~Hz}, \mathrm{H}_{\text {arom. }}$ ), 5.34 (t, 1H, J 9.5 Hz, H-3), 5.17 (d, 1H, J 10.5 Hz, H-1), 4.91 (t, 1H, J 9.5 Hz, H4), 4.68 (t, 1H, J 10.0 Hz, H-2), 4.28-4.19 (m, 2H, H-6a, H-6b), 4.12 (m, 1H, H-5), 4.04 (m, 1H, $\mathrm{NH}_{\mathrm{Fmoc}}$ ), 3.87 (m, 1H, SNH); 3.30 (m, 3H, $\left.\mathrm{CH}_{2 \mathrm{Fmoc}}, \mathrm{CH}_{\mathrm{Fmoc}}\right), 2.00,1.99,1.98,1.94(4 \times \mathrm{s}, 12 \mathrm{H}$, $\left.\mathrm{OCOCH}_{3}\right), 1.72\left(\mathrm{~m}, 2 \mathrm{H}, \varepsilon-\mathrm{CH}_{2}\right), 1.59\left(\mathrm{~m}, 2 \mathrm{H}, \delta-\mathrm{CH}_{2}\right), 1.28\left(\mathrm{~m}, 3 \mathrm{H}, \alpha-\mathrm{CH}, \beta-\mathrm{CH}_{2}\right), 0.85(\mathrm{~m}, 2 \mathrm{H}$, $\left.\gamma-\mathrm{CH}_{2}\right) ;{ }^{13} \mathrm{C}$ NMR (125 MHz, DMSO- $\left.d_{6}\right): \delta 172.5\left(\mathrm{CO}_{2} \mathrm{H}\right), 172.5(\mathrm{OCONH}), 170.5,170.0,169.7$, $169.5\left(4 \times \mathrm{OCOCH}_{3}\right), 143.0,139.9,137.9,135.8,129.9,129.4,129.4,127.7,127.7,121.8,121.8$, 120.5, $120.5\left(13 \times \mathrm{C}_{\mathrm{Fmoc}}\right)$; $110.2(\mathrm{C}-1), 74.7$ (C-5), 73.5 (C-3); 68.4 (C-2 or C-4); 68.2 (C-2 or C4); 62.4 (C-6), $54.6(\alpha-\mathrm{CH}) ; 52.1\left(\mathrm{CH}_{2 \mathrm{Fmoc}}\right) ; 40.4,21.1,21.1,21.1\left(4 \times \mathrm{CH}_{2}\right) ; 20.9,20.8,20.8$, $20.7\left(4 \times \mathrm{OCOCH}_{3}\right) ; \mathrm{LRMS}\left(\mathrm{ESI}^{+}\right): m / z 731[\mathrm{M}+\mathrm{H}]^{+}$.

\subsection{Methyl $N^{\alpha}$-acetyl- $N^{\varepsilon}$-[(2,3,4,6-tetra- $O$-acetyl- $\beta$-D-glucosyl)thio]-L-lysinate (9a)}

The title compound 9a was prepared from $N^{\alpha}$-acetyl-L-lysine methyl ester according to the general procedure 1, using 1.0 equiv. of lysine derivative and 2.3 equiv. of DIPEA. A purification by flash chromatography (4:1 EtOAc/hexane) afforded the title compound 9a as a colorless oil (24\%): $\mathrm{R}_{f} 0.28$ (4:1 EtOAc/hexane); ${ }^{1} \mathrm{H}$ NMR (500 MHz, DMSO- $\left.d_{6}\right): \delta 8.18(\mathrm{~d}, 1 \mathrm{H}$, $J 7.5 \mathrm{~Hz}, \mathrm{NHAc}$ ), 5.32 (t, $1 \mathrm{H}, J 9.5 \mathrm{~Hz}, \mathrm{H}-3$ ), 4.87 (t, 1H, $J 9.5 \mathrm{~Hz}, \mathrm{H}-4), 4.86$ (t, 1H, J 9.5 Hz, H-2), 4.68 (d, 1H, J 10.0 Hz, H-1), 4.18 (td, 1H, J 8.0, 5.0 Hz, $\alpha-\mathrm{CH}), 4.12$ (dd, 1H, $J$ 12.5, 5.0 Hz, H-6a), 4.04 (dd, 1H, J 12.5, 2.0 Hz, H-6b), 3.98 (ddd, 1H, J 10.0, 5.0, 2.5 Hz, H-5), 3.75 (t, $1 \mathrm{H}, J 5.5 \mathrm{~Hz}, \mathrm{SNH}), 3.61\left(\mathrm{~s}, 3 \mathrm{H}, \mathrm{OCH}_{3}\right), 2.79\left(\mathrm{~m}, 2 \mathrm{H}, \varepsilon-\mathrm{CH}_{2}\right), 2.01,2.00,1.99,1.94(4 \times \mathrm{s}, 12 \mathrm{H}$, $\left.\mathrm{OCOCH}_{3}\right), 1.84\left(\mathrm{~s}, 3 \mathrm{H}, \mathrm{NHCOCH}_{3}\right), 1.60\left(\mathrm{~m}, 2 \mathrm{H}, \beta-\mathrm{CH}_{2}\right), 1.40\left(\mathrm{~m}, 2 \mathrm{H}, \delta-\mathrm{CH}_{2}\right), 1.26(\mathrm{~m}, 2 \mathrm{H}, \gamma-$ $\left.\mathrm{CH}_{2}\right) ;{ }^{13} \mathrm{C}$ NMR (125 MHz, DMSO- $\left.d_{6}\right): \delta 172.8\left(\mathrm{CO}_{2} \mathrm{CH}_{3}\right), 170.0,169.5,169.4,169.3,169.3$ $\left(\mathrm{NHCOCH}_{3}, 4 \times \mathrm{OCOCH}_{3}\right), 87.5(\mathrm{C}-1), 74.2(\mathrm{C}-5), 73.3(\mathrm{C}-3), 68.1(\mathrm{C}-4), 67.6(\mathrm{C}-2), 62.0(\mathrm{C}-$ 6), $52.6\left(\varepsilon-\mathrm{CH}_{2}\right), 51.9(\alpha-\mathrm{CH}), 51.7\left(\mathrm{CO}_{2} \mathrm{CH}_{3}\right), 30.8\left(\beta-\mathrm{CH}_{2}\right), 29.2\left(\delta-\mathrm{CH}_{2}\right), 22.6\left(\gamma-\mathrm{CH}_{2}\right), 22.2$, 20.5, 20.5, 20.3, $20.3\left(\mathrm{NHCOCH}_{3}, 4 \times \mathrm{OCOCH}_{3}\right)$; LRMS $\left(\mathrm{ESI}^{+}\right): \mathrm{m} / z 565[\mathrm{M}+\mathrm{H}]^{+}, 587[\mathrm{M}+$ $\mathrm{Na}]^{+}$. HRMS: Calcd for $\mathrm{C}_{23} \mathrm{H}_{36} \mathrm{~N}_{2} \mathrm{O}_{12} \mathrm{SNa}[\mathrm{M}+\mathrm{Na}]^{+}$565.2062, Found 565.2084.

\subsection{Methyl $N$-[(2,3,4,6-tetra- $O$-acetyl- $\beta$-D-glucosyl)sulfonyl]-glycinate (2b)}

Derivative $\mathbf{2 b}$ was prepared by oxidation of compound 2a according to the general procedure 2 . After purification by flash chromatography (3:2 EtOAc/hexane) expected compound $\mathbf{2 b}$ was 
obtained as a white solid (87\%): $\mathrm{mp} 123-124{ }^{\circ} \mathrm{C}$; $[\alpha]_{\mathrm{D}}{ }^{25}-25\left(c\right.$ 1.0, $\left.\mathrm{CHCl}_{3}\right) ; \mathrm{R}_{f} 0.22(1: 1$ EtOAc/hexane); ${ }^{1} \mathrm{H}$ NMR (500 MHz, DMSO- $d_{6}$ ): $\delta 8.02$ (br s, $\left.1 \mathrm{H}, \mathrm{NH}\right), 5.39$ (t, $1 \mathrm{H}, J 9.5 \mathrm{~Hz}, \mathrm{H}-$ 3), 5.28 (t, 1H, J 9.5 Hz, H-2), 4.93 (d, 1H, J 9.5 Hz, H-1), 4.91 (t, 1H, J 9.5 Hz, H-4), 4.15-4.10

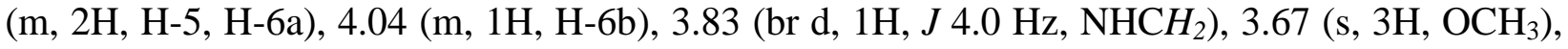
$2.02,1.99,1.96,1.95\left(4 \times \mathrm{s}, 12 \mathrm{H}, \mathrm{OCOCH}_{3}\right) ;{ }^{13} \mathrm{C} \mathrm{NMR}\left(125 \mathrm{MHz}, \mathrm{DMSO}-d_{6}\right): \delta 170.0$ $\left(\mathrm{CO}_{2} \mathrm{CH}_{3}\right), 169.9,169.5,169.2,168.7\left(4 \times \mathrm{OCOCH}_{3}\right), 86.8(\mathrm{C}-1), 74.4(\mathrm{C}-5), 72.6(\mathrm{C}-3), 67.5$ (C-4), 67.4 (C-2), 61.6 (C-6), $51.9\left(\mathrm{CO}_{2} \mathrm{CH}_{3}\right), 44.3\left(\mathrm{NHCH}_{2}\right), 20.4,20.4,20.3,20.2(4 \times$ $\left.\mathrm{OCOCH}_{3}\right) ; \operatorname{LRMS}\left(\mathrm{ESI}^{+}\right): m / z 501\left[\mathrm{M}+\mathrm{NH}_{4}\right]^{+}, 506[\mathrm{M}+\mathrm{Na}]^{+}$; HRMS: Calcd for $\mathrm{C}_{17} \mathrm{H}_{25} \mathrm{NO}_{13} \mathrm{SNa}[\mathrm{M}+\mathrm{Na}]^{+}$506.0939, Found 506.0929.

\subsection{Methyl $N$-[(2,3,4,6-tetra- $O$-acetyl- $\beta$-D-glucosyl)sulfonyl]-L-valinate $\quad(3 \mathrm{~b})$}

Glycoconjugate $\mathbf{3 b}$ was prepared by oxidation of compound 3a according to the general procedure 2. After purification by flash chromatography (2:3 then 1:1 EtOAc/hexane) expected compound 3b was obtained as a white solid (78\%): mp 104-105 ${ }^{\circ} \mathrm{C} ;[\alpha]_{\mathrm{D}}{ }^{25}-30\left(c 1.0, \mathrm{CHCl}_{3}\right) ; \mathrm{R}_{f}$

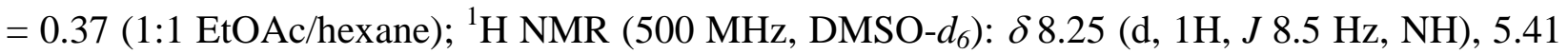
(t, 1H, J 9.5 Hz, H-3), 5.17 (t, 1H, J 9.5 Hz, H-2), 4.94 (d, 1H, J 9.5 Hz, H-1), 4.91 (t, 1H, J 9.5 Hz, H-4), 4.20 (dd, 1H, J 4.5, 12.5 Hz, H-6a), 4.09 (ddd, 1H, J 2.5, 4.5, 10.0 Hz, H-5), 3.91 (dd, $1 \mathrm{H}, J$ 2.5, $12.5 \mathrm{~Hz}, \mathrm{H}-6 \mathrm{~b}), 3.25$ (dd, 1H, J 6.5, 8.5 Hz, NHCH), 3.66 (s, 3H, $\mathrm{OCH}_{3}$ ), 2.01 (s, 3H, $\left.\mathrm{OCOCH}_{3}\right), 1.99\left(\mathrm{~m}, 1 \mathrm{H}, \mathrm{CH}_{3} \mathrm{CHCH}_{3}\right), 1.98,1.95,1.94\left(3 \times \mathrm{s}, 9 \mathrm{H}, \mathrm{OCOCH}_{3}\right), 0.89(\mathrm{~d}, 3 \mathrm{H}, J 7.0$ $\mathrm{Hz}, \mathrm{CH}_{3} \mathrm{CHCH}_{3}$ ); 0.88 (d, 3H, $\left.J .0 \mathrm{~Hz}, \mathrm{CH}_{3} \mathrm{CHCH}_{3}\right) ;{ }^{13} \mathrm{C}$ NMR (125 MHz, DMSO- $\left.d_{6}\right): \delta 171.7$ $\left(\mathrm{CO}_{2} \mathrm{CH}_{3}\right), 170.0,169.5,169.1,168.6\left(4 \times \mathrm{OCOCH}_{3}\right), 85.6(\mathrm{C}-1), 74.4(\mathrm{C}-5), 72.6(\mathrm{C}-3), 67.4$ (C-4), 67.3 (C-2), 61.8 (NHCH), 61.4 (C-6), $51.8\left(\mathrm{CO}_{2} \mathrm{CH}_{3}\right), 30.5\left(\mathrm{CH}_{3} \mathrm{CHCH}_{3}\right), 20.4,20.4,20.3$, $20.2\left(4 \times \mathrm{OCOCH}_{3}\right), 18.8,17.9\left(\mathrm{CH}_{3} \mathrm{CHCH}_{3}\right) ; \mathrm{LRMS}\left(\mathrm{ESI}^{+}\right): \mathrm{m} / z 543\left[\mathrm{M}+\mathrm{NH}_{4}\right]^{+}, 548[\mathrm{M}+$ $\mathrm{Na}]^{+}$; HRMS: Calcd for $\mathrm{C}_{20} \mathrm{H}_{31} \mathrm{~N}_{1} \mathrm{O}_{13} \mathrm{SNa}[\mathrm{M}+\mathrm{Na}]^{+}$548.1408, Found 548.1407.

\subsection{Methyl $N$-[(2,3,4,6-tetra- $O$-acetyl- $\beta$-D-glucosyl)sulfonyl $]-R$-phenylglycinate (4b)}

Glycoconjugate $\mathbf{4 b}$ was prepared by oxidation of compound $\mathbf{4 a}$ according to the general procedure 2. After purification by flash chromatography (3:2 EtOAc/hexane) expected compound 4b was obtained as a white solid (87\%): mp 204-205 ${ }^{\circ} \mathrm{C}$; $[\alpha]_{\mathrm{D}}{ }^{25}-100\left(c 1.0, \mathrm{CHCl}_{3}\right)$; $\mathrm{R}_{f} 0.37$ (1:1 EtOAc/hexane); ${ }^{1} \mathrm{H}$ NMR (500 MHz, DMSO-d $\left.d_{6}\right): \delta 8.88(\mathrm{~d}, 1 \mathrm{H}, J 9.0 \mathrm{~Hz}, \mathrm{NH})$, 7.42-7.34 (m, 5H, Harom.), 5.38 (t, 1H, J 9.5 Hz, H-3), 5.22 (t, 1H, J 9.5 Hz, H-2), 5.12 (d, 1H, J 
$9.0 \mathrm{~Hz}, \mathrm{NHCH}), 4.97$ (d, 1H, J 9.5 Hz, H-1), 4.83 (t, 1H, J 9.5 Hz, H-4), 4.15-4.10 (m, 2H, H-5, H-6a), 3.85 (m, 1H, H-6b), 3.68 (s, 3H, $\left.\mathrm{OCH}_{3}\right), 2.00,1.98,1.95,1.93\left(4 \times \mathrm{s}, 12 \mathrm{H}, \mathrm{OCOCH}_{3}\right) ;{ }^{13} \mathrm{C}$ NMR (125 MHz, DMSO- $\left.d_{6}\right): \delta 70.8\left(\mathrm{CO}_{2} \mathrm{CH}_{3}\right), 170.0,169.5,169.2,168.7\left(4 \times \mathrm{OCOCH}_{3}\right), 136.3$ ( $\left.\mathrm{C}_{\text {arom. }}\right), 128.5,128.5,128.3,127.4,127.4$ (5 × $\left.\mathrm{CH}_{\text {arom. }}\right), 86.8$ (C-1), 74.5 (C-5), 72.6 (C-3), 67.6 (C-2), $67.4(\mathrm{C}-4), 61.5(\mathrm{C}-6), 59.9(\mathrm{NHCH}), 52.5\left(\mathrm{CO}_{2} \mathrm{CH}_{3}\right), 20.4,20.4,20.3,20.2(4 \times$ $\left.\mathrm{OCOCH}_{3}\right) ; \operatorname{LRMS}\left(\mathrm{ESI}^{+}\right): m / z 577\left[\mathrm{M}+\mathrm{NH}_{4}\right]^{+}, 582[\mathrm{M}+\mathrm{Na}]^{+}$; HRMS: Calcd for $\mathrm{C}_{23} \mathrm{H}_{29} \mathrm{NO}_{13} \mathrm{SNa}[\mathrm{M}+\mathrm{Na}]^{+}$582.1252, Found 582.1274.

\subsection{Methyl $N$-[(2,3,4,6-tetra- $O$-acetyl- $\beta$-D-glucosyl)sulfonyl]-L-serinate (5b)}

Glycoconjugate 5b was prepared by oxidation of compound 5a according to the general procedure 2. After purification by flash chromatography (3:2 EtOAc/hexane) expected compound $\mathbf{5 b}$ was obtained as a white solid (57\%): mp 179-180 ${ }^{\circ} \mathrm{C} ;[\alpha]_{\mathrm{D}}{ }^{25}-40\left(c 1.0, \mathrm{CHCl}_{3}\right) ; \mathrm{R}_{f}$ 0.22 (1:1 EtOAc/hexane); ${ }^{1} \mathrm{H}$ NMR (500 MHz, DMSO-d $): \delta 8.92$ (br s, $\left.1 \mathrm{H}, \mathrm{NH}\right), 5.37$ (t, $1 \mathrm{H}, J$ $9.5 \mathrm{~Hz}, \mathrm{H}-3), 5.20$ (t, 1H, J 9.5 Hz, H-2), 5.06 (br t, 1H, J $5.5 \mathrm{~Hz}, \mathrm{NHCH}), 4.94$ (d, 1H, J 10.0 Hz, H-1), 4.93 (t, 1H, J 10.5 Hz, H-4), 4.18 (dd, 1H, J 4.5, 12.5 Hz, H-6a), 4.11 (m, 1H, H-5), 4.01 (t, 1H, J $5.0 \mathrm{~Hz}, \mathrm{CH}_{2} \mathrm{OH}$ ), 3.97 (dd, 1H, J 1.5, $\left.12.5 \mathrm{~Hz}, \mathrm{H}-6 \mathrm{~b}\right), 3.67$ (m, 2H, CH $\mathrm{OH}$ ), 3.66 $\left(\mathrm{s}, 3 \mathrm{H}, \mathrm{OCH}_{3}\right), 2.01,1.98,1.95,1.94\left(4 \times \mathrm{s}, 12 \mathrm{H}, \mathrm{OCOCH}_{3}\right) ;{ }^{13} \mathrm{C} \mathrm{NMR}\left(125 \mathrm{MHz}, \mathrm{DMSO}-d_{6}\right): \delta$ $170.7\left(\mathrm{CO}_{2} \mathrm{CH}_{3}\right), 170.1,169.6,169.3,168.9\left(4 \times \mathrm{OCOCH}_{3}\right), 86.2(\mathrm{C}-1), 74.5(\mathrm{C}-5), 72.8(\mathrm{C}-3)$, $67.5(\mathrm{C}-4), 67.4(\mathrm{C}-2), 62.6(\mathrm{NHCH}), 61.4(\mathrm{C}-6), 58.5\left(\mathrm{CH}_{2} \mathrm{OH}\right), 52.1\left(\mathrm{CO}_{2} \mathrm{CH}_{3}\right), 20.5,20.5$, 20.4, $20.3\left(4 \times \mathrm{OCOCH}_{3}\right) ; \mathrm{LRMS}\left(\mathrm{ESI}^{+}\right): \mathrm{m} / z 531\left[\mathrm{M}+\mathrm{NH}_{4}\right]^{+}, 536[\mathrm{M}+\mathrm{Na}]^{+}$; HRMS: Calcd for $\mathrm{C}_{18} \mathrm{H}_{27} \mathrm{NO}_{14} \mathrm{SNa}[\mathrm{M}+\mathrm{Na}]^{+}$536.1044, Found 536.1054.

\subsection{Methyl $N$-[(2,3,4,6-tetra- $O$-acetyl- $\beta$-D-glucosyl)sulfonyl]-L-prolinate (6b)}

Glycoconjugate $\mathbf{6 b}$ was prepared by oxidation of compound $\mathbf{6 a}$ according to the general procedure 2. After purification by flash chromatography (1:1 EtOAc/hexane) expected compound $\mathbf{6 b}$ was obtained as a white solid (64\%): mp 181-182 ${ }^{\circ} \mathrm{C}$; $[\alpha]_{\mathrm{D}}{ }^{25}-58$ (c 1.0, $\left.\mathrm{CHCl}_{3}\right) ; \mathrm{R}_{f}$ 0.32 (1:1 EtOAc/hexane); ${ }^{1} \mathrm{H}$ NMR (500 MHz, DMSO-d $): \delta 5.31$ (d, $\left.1 \mathrm{H}, J 9.0 \mathrm{~Hz}, \mathrm{H}-1\right), 5.29$ (t, 1H, J $9.5 \mathrm{~Hz}, \mathrm{H}-3), 5.26$ (t, 1H, J $9.0 \mathrm{~Hz}, \mathrm{H}-2), 4.94$ (t, 1H, J 9.5 Hz, H-4), 4.41 (dd, 1H, J 4.0,

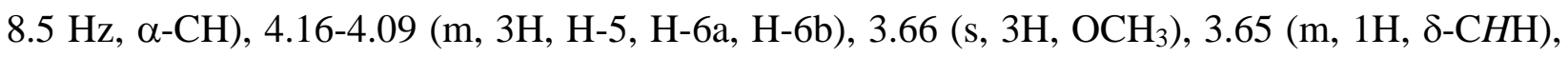
$3.41(\mathrm{~m}, 1 \mathrm{H}, \delta-\mathrm{CH} H) ; 2.28(\mathrm{~m}, 1 \mathrm{H}, \beta-\mathrm{CHH}), 2.02\left(\mathrm{~s}, 6 \mathrm{H}, 2 \times \mathrm{OCOCH}_{3}\right), 2.00\left(\mathrm{~s}, 3 \mathrm{H}, \mathrm{OCOCH}_{3}\right)$, 
$1.95\left(\mathrm{~s}, 3 \mathrm{H}, \mathrm{OCOCH}_{3}\right), 1.92-1.87\left(\mathrm{~m}, 3 \mathrm{H}, \gamma-\mathrm{CH}_{2}, \beta-\mathrm{CH} H\right) ;{ }^{13} \mathrm{C}$ NMR $\left(125 \mathrm{MHz}\right.$, DMSO- $\left.d_{6}\right): \delta$ $172.2\left(\mathrm{CO}_{2} \mathrm{CH}_{3}\right), 169.9,169.5,169.2,168.5\left(4 \times \mathrm{OCOCH}_{3}\right), 85.9(\mathrm{C}-1), 74.8(\mathrm{C}-5), 72.7(\mathrm{C}-3)$, $67.4(\mathrm{C}-4), 67.1(\mathrm{C}-2), 61.8(\mathrm{C}-6), 59.8(\alpha-\mathrm{CH}), 52.1\left(\mathrm{CO}_{2} \mathrm{CH}_{3}\right), 50.1\left(\delta-\mathrm{CH}_{2}\right), 30.9\left(\beta-\mathrm{CH}_{2}\right)$, $24.3\left(\gamma-\mathrm{CH}_{2}\right), 20.4,20.3,20.3,20.2\left(4 \times \mathrm{OCOCH}_{3}\right) ; \mathrm{LRMS}\left(\mathrm{ESI}^{+}\right): \mathrm{m} / z 541\left[\mathrm{M}+\mathrm{NH}_{4}\right]^{+}, 546[\mathrm{M}$ $+\mathrm{Na}]^{+}$; HRMS: Calcd for $\mathrm{C}_{20} \mathrm{H}_{29} \mathrm{NO}_{13} \mathrm{SNa}\left[\mathrm{M}+\mathrm{Na}^{+}\right.$546.1252, Found 546.1234 .

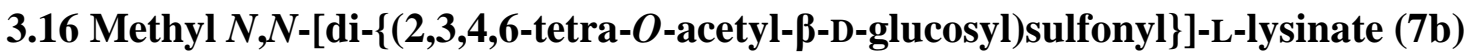

The title compound $\mathbf{7 b}$ was obtained form compound $\mathbf{7 a}$ according to the general procedure 3 . A purification by flash chromatography (2:1 EtOAc/hexane) afforded the title compound $\mathbf{7 b}$ as a white solid (39\%): $\mathrm{mp} 78-80{ }^{\circ} \mathrm{C} ;[\alpha]_{\mathrm{D}}{ }^{25}-31\left(c 1.0, \mathrm{CHCl}_{3}\right) ; \mathrm{R}_{f} 0.24$ (2:1 EtOAc/hexane); ${ }^{1} \mathrm{H}$ NMR $\left(500 \mathrm{MHz}, \mathrm{DMSO}-d_{6}\right): \delta 8.29(\mathrm{~d}, 1 \mathrm{H}, J 8.0 \mathrm{~Hz}, \alpha-\mathrm{NH}), 7.56(\mathrm{t}, 1 \mathrm{H}, J 6.0 \mathrm{~Hz}, \varepsilon-\mathrm{NH}), 5.39(\mathrm{t}, 1 \mathrm{H}$, $J 9.5 \mathrm{~Hz}, \mathrm{H}-3$ or H-3'), 5.38 (t, 1H, J 9.5 Hz, H-3 or H-3'), 5.18 (t, $1 \mathrm{H}, J 9.5 \mathrm{~Hz}, \mathrm{H}-2$ or H-2'), 5.17 (t, $1 \mathrm{H}, J 9.5 \mathrm{~Hz}, \mathrm{H}-2$ or H-2'), 4.93-4.89 (m, 4H, H-1, H-1', H-4, H-4'), 4.22 (dd, 1H, $J$ 13.0, 5.0 Hz, H-6a or H-6a'), 4.20-4.15 (m, 2H, H-5 or H-5', H-6a or H-6a'), 4.11 (ddd, 1H, $J$ 10.0, 4.5, $2.0 \mathrm{~Hz}, \mathrm{H}-5$ or H-5'), 4.03 (br d, 1H, $J 10.5 \mathrm{~Hz}, \mathrm{H}-6 \mathrm{~b}$ or H6b'), 3.92 (dd, 1H, $J$ 12.5, $2.0 \mathrm{~Hz}, \mathrm{H}-6 \mathrm{~b}$ or H6b'), 3.88 (m, 1H, $\alpha-\mathrm{CH}), 3.66$ (s, 3H, $\left.\mathrm{OCH}_{3}\right), 2.98\left(\mathrm{~m}, 2 \mathrm{H}, \varepsilon-\mathrm{CH}_{2}\right), 2.01$ (s, $\left.3 \mathrm{H}, \mathrm{OCOCH}_{3}\right), 1.99\left(\mathrm{~s}, 6 \mathrm{H}, 2 \times \mathrm{OCOCH}_{3}\right), 1.98\left(\mathrm{~s}, 3 \mathrm{H}, \mathrm{OCOCH}_{3}\right), 1.95\left(\mathrm{~s}, 6 \mathrm{H}, 2 \times \mathrm{OCOCH}_{3}\right)$, $1.94\left(\mathrm{~s}, 6 \mathrm{H}, 2 \times \mathrm{OCOCH}_{3}\right), 1.63\left(\mathrm{~m}, 2 \mathrm{H}, \beta-\mathrm{CH}_{2}\right), 1.45\left(\mathrm{~m}, 2 \mathrm{H}, \delta-\mathrm{CH}_{2}\right), 1.34\left(\mathrm{~m}, 2 \mathrm{H}, \gamma-\mathrm{CH}_{2}\right) ;{ }^{13} \mathrm{C}$ NMR (125 MHz, DMSO- $\left.d_{6}\right): \delta 172.1\left(\mathrm{CO}_{2} \mathrm{CH}_{3}\right), 170.0,169.9,169.5,169.5,169.2,169.1,168.6$, $168.5\left(8 \times \mathrm{OCOCH}_{3}\right), 85.9,85.5\left(\mathrm{C}-1, \mathrm{C}-1^{\prime}\right), 74.4,74.3$ (C-5, C5'), 72.8, 72.7 (C-3, C-3'), 67.6, $67.6\left(\mathrm{C}-4, \mathrm{C}-4^{\prime}\right), 67.4,67.3\left(\mathrm{C}-2, \mathrm{C}-2^{\prime}\right), 61.7,61.4\left(\mathrm{C}-6, \mathrm{C}^{-} 6^{\prime}\right), 56.0(\alpha-\mathrm{CH}), 52.0\left(\mathrm{OCH}_{3}\right), 42.7$ $\left(\varepsilon-\mathrm{CH}_{2}\right), 31.8\left(\beta-\mathrm{CH}_{2}\right), 29.3\left(\delta-\mathrm{CH}_{2}\right), 22.1\left(\gamma-\mathrm{CH}_{2}\right), 20.4(\times 4), 20.3,20.3,20.2,20.2(8 \times$ $\mathrm{OCOCH}_{3}$ ); LRMS $\left(\mathrm{ESI}^{+}\right): m / z, 971[\mathrm{M}+\mathrm{Na}]^{+}$; HRMS: Calcd for $\mathrm{C}_{35} \mathrm{H}_{52} \mathrm{~N}_{2} \mathrm{O}_{24} \mathrm{~S}_{2} \mathrm{Na}[\mathrm{M}+\mathrm{Na}]^{+}$ 971.2244, Found 971.2260.

\subsection{7 $N^{\alpha}$-Fluorenylmethyloxycarbonyl- $N^{\varepsilon}$-[(2,3,4,6-tetra- $O$-acetyl- $\beta$-D-glucosyl)sulfonyl]-L- lysine (8b)}

The title compound $\mathbf{8 b}$ was prepared from compound $\mathbf{8 a}$ according to the general procedure 3 . Purification by flash chromatography (3:2 acetone/hexane $+\mathrm{AcOH} 1.4 \% \mathrm{v} / \mathrm{v})$ afforded the title

compound $\mathbf{8 b}$ as a yellow oil $(69 \%):[\alpha]_{\mathrm{D}}{ }^{25}-25\left(c\right.$ 1.0, $\left.\mathrm{CHCl}_{3}\right) ; \mathrm{R}_{f} 0.33$ (3:2 acetone/hexane + 
AcOH 1.4\% v/v); ${ }^{1} \mathrm{H}$ NMR (500 MHz, DMSO- $\left.d_{6}\right): \delta 7.89\left(\mathrm{~d}, 2 \mathrm{H}, J 7.5 \mathrm{~Hz}, \mathrm{H}_{\text {arom. }}\right), 7.72(\mathrm{~m}, 2 \mathrm{H}$, $\mathrm{H}_{\text {arom.) }}$ ), $7.45\left(\mathrm{~m}, 1 \mathrm{H}, \mathrm{SO}_{2} \mathrm{NH}\right), 7.42$ (t, $2 \mathrm{H}, J 7.5 \mathrm{~Hz}, \mathrm{H}_{\text {arom. }}$ ), 7.33 (q, $2 \mathrm{H}, J 7.5 \mathrm{~Hz}, \mathrm{H}_{\text {arom. }}$ ), 3.30 (s, $1 \mathrm{H}, \mathrm{CH}_{\mathrm{Fmoc}}$ ), 5.40 (t, 1H, J 9.0 Hz, H-3), 5.05 (d, 1H, J 10.0 Hz, H-2), 4.96 (t, 1H, J 9.5 Hz, H-4), 4.73 (d, 1H, J 10.0 Hz, H-1), 4.29-4.13 (m, 3H, H-5, H-6a, H-6b), 3.92 (m, 2H, ع-CH $\mathrm{CH}_{2}$, 3.42 (br $\left.\mathrm{s}, 2 \mathrm{H}, \mathrm{CH}_{2 \mathrm{Fmoc}}\right), 3.18(\mathrm{~m}, 1 \mathrm{H}, \mathrm{FmocNH}), 2.02,1.99,1.97,1.94\left(4 \times \mathrm{s}, 12 \mathrm{H}, \mathrm{OCOCH}_{3}\right), 1.75(\mathrm{~m}$, $1 \mathrm{H}, \alpha-\mathrm{CH}), 1.64\left(\mathrm{~m}, 2 \mathrm{H}, \delta-\mathrm{CH}_{2}\right), 1.28\left(\mathrm{~m}, 2 \mathrm{H}, \beta-\mathrm{CH}_{2}\right), 0.84\left(\mathrm{~m}, 2 \mathrm{H}, \gamma-\mathrm{CH}_{2}\right) ;{ }^{13} \mathrm{C}$ NMR $(125$ MHz, DMSO- $\left.d_{6}\right): \delta 172.0\left(\mathrm{CO}_{2} \mathrm{H}\right), 170.2,169.6,169.5,169.2\left(4 \times \mathrm{OCOCH}_{3}\right), 142.6,139.4$, 137.4, 128.9, 128.9, 127.3, 127.3, 121.3, 121.3, $120.0(\times 3), 109.7\left(13 \times \mathrm{C}_{\mathrm{Fmoc}}\right), 90.3(\mathrm{C}-1), 74.4$ (C-5), 72.5 (C-3), 68.4 (C-2 or C-4), 68.2 (C-2 or C-4), $61.4(\mathrm{C}-6), 55.8(\alpha-\mathrm{CH}), 45.6\left(\mathrm{CH}_{2 \mathrm{Fmoc}}\right)$, 30.6, 29.5, 21.1, $21.1\left(4 \times \mathrm{CH}_{2}\right), 20.5,20.3,20.3,20.2\left(4 \times \mathrm{OCOCH}_{3}\right) ; \mathrm{LRMS}\left(\mathrm{ESI}^{+}\right): \mathrm{m} / z 785[\mathrm{M}$ $+\mathrm{Na}^{+}$; $\left(\mathrm{ESI}^{-}\right): m / z=761[\mathrm{M}-\mathrm{H}]^{-}$; HRMS: Calcd for $\mathrm{C}_{35} \mathrm{H}_{42} \mathrm{~N}_{2} \mathrm{O}_{15} \mathrm{SNa}[\mathrm{M}+\mathrm{Na}]^{+}$785.2198, Found 785.2232.

\subsection{Methyl $N^{a}$-acetyl- $N^{\varepsilon}$-[(2,3,4,6-tetra- $O$-acetyl- $\beta$-D-glucosyl)sulfonyl]-L-lysinate (9b)}

The title compound $\mathbf{9 b}$ was obtained from compound $9 \mathbf{a}$ according to the general procedure 3 . Purification by flash chromatography (3:2 acetone/hexane) afforded the title compound $\mathbf{9 b}$ as a colorless oil (33\%): $\mathrm{R}_{f} 0.27$ (3:2 acetone/hexane); ${ }^{1} \mathrm{H}$ NMR (500 MHz, DMSO- $\left.d_{6}\right): \delta 8.33(\mathrm{~d}, 1 \mathrm{H}$, $J 7.0 \mathrm{~Hz}, \mathrm{NHAc}), 7.80$ (t, 1H, J $5.5 \mathrm{~Hz}, \varepsilon-\mathrm{NH}), 5.40$ (t, 1H, J 8.0 Hz, H-3), 5.17 (t, 1H, J 8.0 Hz, H-2), 4.93 (d, 1H, J 8.5 Hz, H-1), 4.92 (t, 1H, J 8.5 Hz, H-4), 4.22 (dd, 1H,J 10.5, 4.0 Hz, H-6a), 4.12 (ddd, 1H, J 8.5, 4.0, 2.0 Hz, H-5), 3.91 (dd, 1H, J 10.5, 2.0 Hz, H-6b), 3.86 (td, 1H, J 7.5, $4.5 \mathrm{~Hz}, \alpha-\mathrm{CH}), 3.65\left(\mathrm{~s}, 3 \mathrm{H}, \mathrm{OCH}_{3}\right), 2.99\left(\mathrm{~m}, 2 \mathrm{H}, \varepsilon-\mathrm{CH}_{2}\right), 2.01,1.98,1.95,1.94(4 \times \mathrm{s}, 12 \mathrm{H}$, $\left.\mathrm{OCOCH}_{3}\right), 1.78\left(\mathrm{~s}, 3 \mathrm{H}, \mathrm{NHCOCH}_{3}\right), 1.61\left(\mathrm{~m}, 2 \mathrm{H}, \beta-\mathrm{CH}_{2}\right), 1.38\left(\mathrm{~m}, 2 \mathrm{H}, \delta-\mathrm{CH}_{2}\right), 1.31(\mathrm{~m}, 2 \mathrm{H}, \gamma-$ $\left.\mathrm{CH}_{2}\right) ;{ }^{13} \mathrm{C}$ NMR (125 MHz, DMSO- $\left.d_{6}\right): \delta 172.4\left(\mathrm{CO}_{2} \mathrm{CH}_{3}\right), 170.3,169.8,169.6,169.5,168.9(4$ $\left.\times \mathrm{OCOCH}_{3}, \mathrm{NHCOCH}_{3}\right), 86.0(\mathrm{C}-1), 74.6(\mathrm{C}-5), 72.9(\mathrm{C}-3), 67.6(\mathrm{C}-2$ or $\mathrm{C}-4), 67.5(\mathrm{C}-2$ or $\mathrm{C}-$ 4), $61.6(\mathrm{C}-6), 56.3(\alpha-\mathrm{CH}), 38.5\left(\varepsilon-\mathrm{CH}_{2}\right)$ 32.5, 28.6, $24.0\left(3 \times \mathrm{CH}_{2}\right), 22.7\left(\mathrm{OCH}_{3}\right) ; 20.6,20.6$, 20.5, 20.4, $20.0\left(4 \times \mathrm{OCOCH}_{3}, \mathrm{NHCOCH}_{3}\right)$; LRMS $\left(\mathrm{ESI}^{+}\right): m / z 598[\mathrm{M}+\mathrm{H}]^{+}, 619[\mathrm{M}+\mathrm{Na}]^{+}$; HRMS: Calcd for $\mathrm{C}_{23} \mathrm{H}_{36} \mathrm{~N}_{2} \mathrm{O}_{14} \mathrm{SNa}[\mathrm{M}+\mathrm{Na}]^{+}$619.1779, Found 619.1790.

\subsection{Methyl $N$-[( $\beta$-D-glucosyl)sulfonyl]-glycinate (2c)}


The title compound $\mathbf{2 c}$ was obtained from the per-acetylated derivative $\mathbf{2 b}$ following the general procedure 3. Lyophilization afforded the title compound $\mathbf{2 c}$ as a hygroscopic slightly yellow solid (94\%): $[\alpha]_{\mathrm{D}}{ }^{25}-11$ (c 1.0, $\left.\mathrm{MeOH}\right) ; \mathrm{R}_{f} 0.43\left(95: 5 \mathrm{CH}_{3} \mathrm{CN} / \mathrm{H}_{2} \mathrm{O}\right) ;{ }^{1} \mathrm{H}$ NMR $(500 \mathrm{MHz}$, DMSO$\left.d_{6}\right): \delta 7.49$ (br s, 1H, NH), $5.13(\mathrm{~m}, 2 \mathrm{H}, \mathrm{OH}-2, \mathrm{OH}-3), 5.03$ (d, 1H, J 4.5 Hz, OH-4), 4.47 (br s, 1H, OH-6), 4.22 (d, 1H, J 9.5 Hz, H-1), 3.85 (m, 2H, NHCH $), 3.67$ (dd, 1H, J 4.5, $11.5 \mathrm{~Hz}, \mathrm{H}-$ 6a), 3.65 (s, 3H, $\mathrm{OCH}_{3}$ ), 3.44 (m, 2H, H-2, H-6b), 3.22 (m, 2H, H-3, H-5), 3.05 (m, 1H, H-4); ${ }^{13} \mathrm{C}$ NMR (125 MHz, DMSO- $\left.d_{6}\right): \delta 170.1\left(\mathrm{CO}_{2} \mathrm{CH}_{3}\right), 90.5$ (C-1), 81.2 (C-5), 77.4 (C-3), $70.4(\mathrm{C}-4)$, $69.5(\mathrm{C}-2), 61.0(\mathrm{C}-6), 51.8\left(\mathrm{OCH}_{3}\right), 51.8\left(\mathrm{NHCH}_{2}\right)$; LRMS $\left(\mathrm{ESI}^{+}\right): \mathrm{m} / z 338\left[\mathrm{M}+\mathrm{Na}^{+}\right.$; $\mathrm{HRMS}$ : Calcd for $\mathrm{C}_{9} \mathrm{H}_{17} \mathrm{NO}_{9} \mathrm{SNa}[\mathrm{M}+\mathrm{Na}]^{+} 338.0516$, Found 338.0512.

\subsection{Methyl $N$-[( $\beta$-D-glucosyl)sulfonyl]-L-valinate (3c)}

The title compound $\mathbf{3} \mathbf{c}$ was obtained from the per-acetylated derivative $\mathbf{3 b}$ following the general procedure 3. Lyophilization afforded the title compound 3c as an off-white solid (93\%): mp 89$93{ }^{\circ} \mathrm{C} ;[\alpha]_{\mathrm{D}}{ }^{25}-31(c$ 1.0, $\mathrm{MeOH}) ; \mathrm{R}_{f} 0.49\left(95: 5 \mathrm{CH}_{3} \mathrm{CN} / \mathrm{H}_{2} \mathrm{O}\right) ;{ }^{1} \mathrm{H}$ NMR (500 MHz, DMSO- $\left.d_{6}\right): \delta$ 7.52 (br s, 1H, NH), 5.14, 5.02 (2 x br s, 3H, OH-2, OH-3, OH-4), 4.37 (br s, 1H, OH-6), 4.14 (d, 1H, J 9.5 Hz, H-1), 3.87 (d, 1H, J 6.0 Hz, NHCH), 3.65 (s, 3H, OCH $), 3.59$ (dd, 1H, J 2.5, 11.5 Hz, H-6a), 3.47 (m, 1H, H-6b), 3.42 (t, 1H, J 9.0 Hz, H-2), 3.21 (t, 1H, J 8.5 Hz, H-3), 3.14 (m, 2H, H-4, H-5), 1.98 (m, 1H, $\left.\mathrm{CH}_{3} \mathrm{CHCH}_{3}\right), 0.89$ (d, 1H, J $\left.6.5 \mathrm{~Hz}, \mathrm{CH}_{3} \mathrm{CHCH}_{3}\right), 0.87$ (d, $1 \mathrm{H}, J 6.5$ $\left.\mathrm{Hz}, \mathrm{CH}_{3} \mathrm{CHCH}_{3}\right) ;{ }^{13} \mathrm{C}$ NMR (125 MHz, DMSO-d $\left.)_{6}\right): \delta 172.0\left(\mathrm{CO}_{2} \mathrm{CH}_{3}\right), 90.5(\mathrm{C}-1), 80.9(\mathrm{C}-5)$, 77.3 (C-3), 70.4 (C-4), 69.1 (C-2), $61.5(\mathrm{C}-6), 60.5(\mathrm{NHCH}), 51.6\left(\mathrm{OCH}_{3}\right) ; 30.8\left(\mathrm{CH}_{3} \mathrm{CHCH}_{3}\right)$, 18.8, $18.0\left(\mathrm{CH}_{3} \mathrm{CHCH}_{3}\right)$; LRMS $\left(\mathrm{ESI}^{+}\right): \mathrm{m} / z 380[\mathrm{M}+\mathrm{Na}]^{+}, 396[\mathrm{M}+\mathrm{K}]^{+}$; HRMS: Calcd for $\mathrm{C}_{12} \mathrm{H}_{23} \mathrm{NO}_{9} \mathrm{SNa}[\mathrm{M}+\mathrm{Na}]^{+}$380.0986, Found 380.0970.

\subsection{Methyl $N$-[( $\beta$-D-glucosyl)sulfonyl]- $R, S$-phenylglycinate $\left(4 \mathrm{c}^{\prime}\right)$}

The title compound $\mathbf{4} \mathbf{c}^{\prime}$ was obtained from the per-acetylated derivative $\mathbf{4 b}$ following the general procedure 3. Lyophilization afforded the title compound $\mathbf{4} \mathbf{c}^{\prime}$ as a hygroscopic white solid (99\%): $[\alpha]_{\mathrm{D}}{ }^{25}-24(c$ 1.0, $\mathrm{MeOH}) ; \mathrm{R}_{f} 0.41\left(95: 5 \mathrm{CH}_{3} \mathrm{CN} / \mathrm{H}_{2} \mathrm{O}\right) ;{ }^{1} \mathrm{H}$ NMR $\left(500 \mathrm{MHz}, \mathrm{DMSO}-d_{6}\right): \delta 8.22$ (br s, $\left.1 \mathrm{H}, \mathrm{NH}_{\mathrm{R}}, \mathrm{N} H_{\mathrm{S}}\right), 7.43-7.33\left(\mathrm{~m}, 5 \mathrm{H}, \mathrm{H}_{\text {arom. }}\right), 5.24,5.22\left(2 \times\right.$ br s, $\left.1 \mathrm{H}, \mathrm{NHCH}_{\mathrm{R}}, \mathrm{NHCH}_{\mathrm{S}}\right), 5.16$ (br s, 0.6H, OH-3 ${ }_{\mathrm{R}}$ ), 5.10 (br s, 0.4H, OH-3s), 5.07 (d, 0.4H, J $5.5 \mathrm{~Hz}, \mathrm{OH}-2_{\mathrm{S}}$ ), 5.03 (d, 0.6H, $J$ $\left.5.5 \mathrm{~Hz}, \mathrm{OH}-4_{\mathrm{R}}\right), 4.98$ (d, 0.4H, J $\left.6.5 \mathrm{~Hz}, \mathrm{OH}-4_{\mathrm{S}}\right), 4.97$ (d, 0.6H, J 6.5 Hz, OH-2 $\left.{ }_{\mathrm{R}}\right), 4.46$ (d, 0.6H, $\left.J 5.5 \mathrm{~Hz}, \mathrm{OH}-6_{\mathrm{R}}\right), 4.44\left(\mathrm{~d}, 0.4 \mathrm{H}, J 6.5 \mathrm{~Hz}, \mathrm{OH}-6_{\mathrm{S}}\right), 4.20\left(\mathrm{~d}, 0.6 \mathrm{H}, J 9.5 \mathrm{~Hz}, \mathrm{H}-1_{\mathrm{R}}\right), 3.81(\mathrm{~d}, 0.4 \mathrm{H}$, 
$\left.J 9.0 \mathrm{~Hz}, \mathrm{H}-1_{\mathrm{S}}\right), 3.64$ (s, 3H, $\left.\mathrm{OCH}_{3}\right), 3.61$ (dd, 0.6H, J 12.0, $\left.6.0 \mathrm{~Hz}, \mathrm{H}-6 \mathrm{a}_{\mathrm{R}}\right), 3.54$ (m, 0.4H, H$\left.6 \mathrm{a}_{\mathrm{S}}\right), 3.51\left(\mathrm{td}, 0.6 \mathrm{H}, J\right.$ 8.0, $\left.5.0 \mathrm{~Hz}, \mathrm{H}-2_{\mathrm{R}}\right), 3.42\left(\mathrm{~m}, 1 \mathrm{H}, \mathrm{H}-6 \mathrm{~b}_{\mathrm{R}}, \mathrm{H}-6 \mathrm{~b}_{\mathrm{S}}\right), 3.35(\mathrm{td}, 0.4 \mathrm{H}, J$ 8.5, 5.0 Hz, H-2 ), 3.23 (br t, 0.6H, J 8.5 Hz, H-3 ${ }_{\mathrm{R}}$ ), 3.19 (m, 0.6H, H-5 $), 3.09$ (m, 0.4H, H-3 $), 3.06$ (m, $\left.0.6 \mathrm{H}, \mathrm{H}-4_{\mathrm{R}}\right), 3.01\left(\mathrm{td}, 0.4 \mathrm{H}, J\right.$ 9.0, $\left.4.0 \mathrm{~Hz}, \mathrm{H}-4_{\mathrm{S}}\right), 2.90\left(\mathrm{~m}, 0.4 \mathrm{H}, \mathrm{H}-5_{\mathrm{S}}\right) ;{ }^{13} \mathrm{C} \mathrm{NMR}(125 \mathrm{MHz}$, DMSO-d $\left.d_{6}\right): \delta 171.0,170.6\left(\mathrm{CO}_{2} \mathrm{CH}_{3}\right), 136.8,128.6,128.1,127.6,127.4\left(5 \times \mathrm{C}_{\text {arom. }}\right), 91.2\left(\mathrm{C}-1_{\mathrm{R}}\right)$, $90.4\left(\mathrm{C}-1_{\mathrm{S}}\right), 81.2\left(\mathrm{C}-5_{\mathrm{R}}\right), 80.8\left(\mathrm{C}-5_{\mathrm{S}}\right), 77.2\left(\mathrm{C}-3_{\mathrm{R}}\right), 77.2\left(\mathrm{C}-3_{\mathrm{S}}\right), 70.2\left(\mathrm{C}-2_{\mathrm{R}}\right), 70.2\left(\mathrm{C}-2_{\mathrm{S}}\right), 69.2$, $69.1\left(\mathrm{C}-4_{\mathrm{R}}, \mathrm{C}-4_{\mathrm{S}}\right), 60.7,60.5\left(\mathrm{C}-6_{\mathrm{R}}, \mathrm{C}-6_{\mathrm{S}}\right), 59.6\left(\mathrm{NHCH}_{\mathrm{R}}\right), 59.6\left(\mathrm{NHCH}_{\mathrm{S}}\right), 52.4,52.3\left(\mathrm{OCH}_{3 \mathrm{R}}\right.$, $\left.\mathrm{OCH}_{3 \mathrm{~S}}\right) ; \operatorname{LRMS}\left(\mathrm{ESI}^{+}\right): m / z 414\left[\mathrm{M}+\mathrm{Na}^{+} ; \mathrm{LRMS}\left(\mathrm{ESI}^{-}\right): m / z=390[\mathrm{M}-\mathrm{H}]^{-}\right.$.

\subsection{Methyl $N$-[( $\beta$-D-glucosyl)sulfonyl]- $R$-phenylglycinate (4c)}

The title compound $\mathbf{4 c}$ was obtained from compound $\mathbf{4 b}$ according to the general procedure 4 . Purification by flash chromatography $\left(85: 15 \mathrm{CH}_{2} \mathrm{Cl}_{2} / \mathrm{MeOH}\right)$ afforded the title compound $\mathbf{4 c}$ as a hygroscopic white solid (23\%): $\mathrm{R}_{f} 0.16$ (85:15 $\left.\mathrm{CH}_{2} \mathrm{Cl}_{2} / \mathrm{MeOH}\right) ;{ }^{1} \mathrm{H}$ NMR (500 MHz, DMSO- $\left.d_{6}\right)$ : $\delta 8.20$ (br s, $1 \mathrm{H}, \mathrm{NH}), 7.42-7.32$ (m, 5H, $\mathrm{H}_{\text {arom. }}$ ), 5.23 (br s, $1 \mathrm{H}, \mathrm{NHCH}$ ), 5.15 (br s, 1H, OH-3), 5.02 (d, 1H, J 5.5 Hz, OH-4), 4.98 (br s, 1H, OH-2), 4.46 (d, 1H, J 5.5 Hz, OH-6), 4.18 (d, 1H, J $9.0 \mathrm{~Hz}, \mathrm{H}-1$ ), 3.63 (s, 3H, $\mathrm{OCH}_{3}$ ), 3.61 (dd, 1H, J 12.0, 6.0 Hz, H-6a), 3.50 (t, 1H, J 9.5 Hz, H-2), 3.42 (m, 1H, H-6b), 3.23 (br t, 1H, J 8.5 Hz, H-3), 3.18 (m, 1H, H-5), 3.07 (td, 1H, J 9.5, 4.0 Hz, $\mathrm{H}-4) ;{ }^{13} \mathrm{C}$ NMR $\left(125 \mathrm{MHz}, \mathrm{DMSO}-d_{6}\right): \delta 170.9\left(\mathrm{CO}_{2} \mathrm{CH}_{3}\right), 129.0,128.5,128.5,128.1,127.4(5 \times$ $\mathrm{C}_{\text {arom.), }} 91.1$ (C-1), 81.1 (C-5), 77.1 (C-3), 70.2 (C-2), 69.1 (C-4), 60.6 (C-6), 59.6 (NHCH), 52.3 $\left(\mathrm{OCH}_{3}\right)$; LRMS $\left(\mathrm{ESI}^{+}\right): \mathrm{m} / z 414[\mathrm{M}+\mathrm{Na}]^{+}$; HRMS: Calcd for $\mathrm{C}_{15} \mathrm{H}_{21} \mathrm{NO}_{9} \mathrm{SNa}[\mathrm{M}+\mathrm{Na}]^{+}$ 414.0829, Found 414.0829.

\subsection{Methyl $N$-[( $\beta$-D-glucosyl)sulfonyl]-L,D-serinate $\left(5 \mathrm{c}^{\prime}\right)$}

The title compound $\mathbf{5} \mathbf{c}^{\prime}$ was obtained from the per-acetylated derivative $\mathbf{5 b}$ following the general procedure 3. Lyophilization afforded the title compound $\mathbf{5} \mathbf{c}^{\prime}$ as a hygroscopic slightly yellow solid (99\%): $[\alpha]_{\mathrm{D}}{ }^{25}-27$ (c 1.0, $\left.\mathrm{MeOH}\right) ; \mathrm{R}_{f} 0.21\left(95: 5 \mathrm{CH}_{3} \mathrm{CN} / \mathrm{H}_{2} \mathrm{O}\right) ;{ }^{1} \mathrm{H}$ NMR $(500 \mathrm{MHz}, \mathrm{DMSO}-$ $\left.d_{6}\right): \delta 7.49\left(\mathrm{~d}, 0.2 \mathrm{H}, J 8.5 \mathrm{~Hz}, \mathrm{NH}_{\mathrm{D}}\right), 7.40\left(\mathrm{~d}, 0.8 \mathrm{H}, J 8.5 \mathrm{~Hz}, \mathrm{NH}_{\mathrm{L}}\right), 5.03(\mathrm{br} \mathrm{m}, 3.2 \mathrm{H}, \mathrm{OH}), 4.52$ (br m, 0.8H, OH), 4.23 (d, 0.8H, J $9.5 \mathrm{~Hz}, \mathrm{H}-1_{\mathrm{L}}$ ), 4.14 (d, 0.2H, J $\left.9.5 \mathrm{~Hz}, \mathrm{H}-1_{\mathrm{D}}\right), 4.08$ (m, 1H, $\mathrm{NHCH}$ ), 3.66 (s, 3H, OCH 3 ), 3.63 (m, 3H, H-6a, $\mathrm{CH}_{2} \mathrm{OH}$ ), 3.47-3.42 (m, 2H, H-2, H-6b), 3.23 (t, $\left.0.8 \mathrm{H}, J 9.0 \mathrm{~Hz}, \mathrm{H}-3_{\mathrm{L}}\right), 3.22\left(\mathrm{t}, 0.2 \mathrm{H}, J 9.0 \mathrm{~Hz}, \mathrm{H}-3_{\mathrm{D}}\right), 3.21\left(\mathrm{~m}, 1 \mathrm{H}, \mathrm{H}-5_{\mathrm{L}}, \mathrm{H}-5_{\mathrm{D}}\right), 3.08$ (t, $0.8 \mathrm{H}, J$ $\left.9.0 \mathrm{~Hz}, \mathrm{H}-4_{\mathrm{L}}\right), 3.03\left(\mathrm{t}, 0.2 \mathrm{H}, J 9.0 \mathrm{~Hz}, \mathrm{H}-4_{\mathrm{D}}\right) ;{ }^{13} \mathrm{C}$ NMR $\left(125 \mathrm{MHz}, \mathrm{DMSO}-d_{6}\right): \delta 171.2$ 
$\left(\mathrm{CO}_{2} \mathrm{CH}_{3 \mathrm{D}}\right), 171.1\left(\mathrm{CO}_{2} \mathrm{CH}_{3 \mathrm{~L}}\right), 90.9\left(\mathrm{C}-1_{\mathrm{D}}\right), 90.1\left(\mathrm{C}-1_{\mathrm{L}}\right), 81.3\left(\mathrm{C}-5_{\mathrm{D}}\right), 81.0\left(\mathrm{C}-5_{\mathrm{L}}\right), 77.4\left(\mathrm{C}-3_{\mathrm{L}}\right)$, $77.3\left(\mathrm{C}-3_{\mathrm{D}}\right), 70.6\left(\mathrm{C}-2_{\mathrm{L}}\right), 70.4\left(\mathrm{C}-2_{\mathrm{D}}\right), 69.6\left(\mathrm{C}-4_{\mathrm{D}}\right), 69.5\left(\mathrm{C}-4_{\mathrm{L}}\right), 62.6\left(\mathrm{NHCH}_{\mathrm{D}}\right), 62.5\left(\mathrm{NHCH}_{\mathrm{L}}\right)$, $61.1\left(\mathrm{C}-6_{\mathrm{D}}\right), 60.9\left(\mathrm{C}-6_{\mathrm{L}}\right), 58.4\left(\mathrm{CH}_{2} \mathrm{OH}_{\mathrm{D}}\right), 58.3\left(\mathrm{CH}_{2} \mathrm{OH}_{\mathrm{L}}\right), 52.1\left(\mathrm{OCH}_{3 \mathrm{D}}\right), 52.1\left(\mathrm{OCH}_{3 \mathrm{~L}}\right) ; \mathrm{LRMS}$ $\left(\mathrm{ESI}^{+}\right): m / z=368[\mathrm{M}+\mathrm{Na}]^{+}, 384[\mathrm{M}+\mathrm{K}]^{+} ;$LRMS (ESI $), m / z=344[\mathrm{M}-\mathrm{H}]^{-}$.

\subsection{Methyl $N$-[( $\beta$-D-glucosyl)sulfonyl]-L-serinate (5c)}

The title compound $\mathbf{5} \mathbf{c}$ was obtained from compound $\mathbf{5 b}$ according to the general procedure 4 . Purification by flash chromatography $\left(9: 1 \mathrm{CH}_{2} \mathrm{Cl}_{2} / \mathrm{MeOH}\right)$ afforded the title compound $\mathbf{5 c}$ as a hygroscopic white solid (36\%): $\mathrm{R}_{f} 0.43\left(85: 15 \mathrm{CH}_{2} \mathrm{Cl}_{2} / \mathrm{MeOH}\right) ;{ }^{1} \mathrm{H}$ NMR (500 MHz, DMSO- $d_{6}$ ): $\delta 7.40(\mathrm{~d}, 1 \mathrm{H}, J 8.0 \mathrm{~Hz}, \mathrm{NH}), 4.23(\mathrm{~d}, 1 \mathrm{H}, J 9.5 \mathrm{~Hz}, \mathrm{H}-1), 4.08(\mathrm{~m}, 1 \mathrm{H}, \mathrm{NHCH}), 3.66(\mathrm{~s}, 3 \mathrm{H}$, $\left.\mathrm{OCH}_{3}\right), 3.63$ (m, 3H, H-6a, $\left.\mathrm{CH}_{2} \mathrm{OH}\right), 3.47-3.42(\mathrm{~m}, 2 \mathrm{H}, \mathrm{H}-2, \mathrm{H}-6 \mathrm{~b}), 3.23$ (t, $\left.1 \mathrm{H}, J 9.0 \mathrm{~Hz}, \mathrm{H}-3\right)$, 3.20 (m, 1H, H-5), 3.08 (t, $1 \mathrm{H}, J 9.0 \mathrm{~Hz}, \mathrm{H}-4) ;{ }^{13} \mathrm{C}$ NMR (125 MHz, DMSO- $\left.d_{6}\right): \delta 171.0\left(\mathrm{OCH}_{3}\right)$; 90.0 (C-1); 81.0 (C-5); 77.4 (C-3); 70.5 (C-2); 69.4 (C-4); 62.4 (NHCH); 60.8 (C-3); 58.3 $\left(\mathrm{CH}_{2} \mathrm{OH}\right) ; 52.0\left(\mathrm{OCH}_{3}\right)$ assignments were confirmed by ${ }^{1} \mathrm{H}^{-13} \mathrm{C}$ HSQC; LRMS $\left(\mathrm{ESI}^{+}\right)$: $\mathrm{m} / z 368$ $[\mathrm{M}+\mathrm{Na}]^{+}$; HRMS: Calcd for $\mathrm{C}_{10} \mathrm{H}_{19} \mathrm{NO}_{10} \mathrm{SNa}[\mathrm{M}+\mathrm{Na}]^{+}$368.0622, Found 368.0606.

\subsection{Methyl $N$-[( $\beta$-D-glucosyl)sulfonyl]-L-prolinate (6c)}

The title compound $\mathbf{6 c}$ was obtained from the per-acetylated derivative $\mathbf{6 b}$ following the general procedure 3. Lyophilization afforded the title compound $\mathbf{6 c}$ as a hygroscopic slightly yellow solid (88\%): $[\alpha]_{\mathrm{D}}{ }^{25}-87(c 1.0, \mathrm{MeOH}) ; \mathrm{R}_{f} 0.41\left(95: 5 \mathrm{CH}_{3} \mathrm{CN} / \mathrm{H}_{2} \mathrm{O}\right) ;{ }^{1} \mathrm{H}$ NMR $(500 \mathrm{MHz}$, DMSO$\left.d_{6}\right): \delta 5.27(\mathrm{~d}, 1 \mathrm{H}, J 6.0 \mathrm{~Hz}, \mathrm{OH}-2), 5.11(\mathrm{~d}, 1 \mathrm{H}, J 5.0 \mathrm{~Hz}, \mathrm{OH}-3), 5.03(\mathrm{~d}, 1 \mathrm{H}, J 5.5 \mathrm{~Hz}, \mathrm{OH}-4)$, 4.55 (d,1H, J 9.5 Hz, H-1), 4.54 (t, 1H, J 5.5 Hz, OH-6), 4.42 (dd, 1H, J 8.5, 3.5 Hz, $\alpha-C H), 3.71$ (dd, 1H, J 10.0, 4.5 Hz, H-6a), 3.65 (s, 3H, $\mathrm{OCH}_{3}$ ), 3.46-3.41 (m, 2H, H-2, H-6b), 3.37 (m, 1H, H-5), 3.29 (m, 2H, $\delta-\mathrm{CH}_{2}$ ), 3.23 (td, $1 \mathrm{H}, J 8.5,5.0 \mathrm{~Hz}, \mathrm{H}-3$ ), 3.07 (td, $1 \mathrm{H}, J$ 9.0, $5.5 \mathrm{~Hz}, \mathrm{H}-4$ ), $2.29(\mathrm{~m}, 1 \mathrm{H}, \beta-\mathrm{CH} H), 1.96(\mathrm{~m}, 1 \mathrm{H}, \beta-\mathrm{CHH}), 1.89-1.78\left(\mathrm{~m}, 2 \mathrm{H}, \gamma-\mathrm{CH}_{2}\right) ;{ }^{13} \mathrm{C} \mathrm{NMR}(125 \mathrm{MHz}$, DMSO- $\left.d_{6}\right): \delta 172.8\left(\mathrm{CO}_{2} \mathrm{CH}_{3}\right), 89.4(\mathrm{C}-1), 81.2(\mathrm{C}-5), 77.6$ (C-3), $70.4(\mathrm{C}-4), 69.3(\mathrm{C}-2), 60.8$ (C-6), $59.5(\alpha-\mathrm{CH}), 51.9\left(\mathrm{OCH}_{3}\right), 49.6\left(\delta-\mathrm{CH}_{2}\right), 30.8\left(\beta-\mathrm{CH}_{2}\right), 24.1\left(\gamma-\mathrm{CH}_{2}\right)$; LRMS $\left(\mathrm{ESI}^{+}\right): \mathrm{m} / \mathrm{z}$ $378[\mathrm{M}+\mathrm{Na}]^{+}$; LRMS (ESI): $m / z 390[\mathrm{M}+\mathrm{Cl}]^{-}$; HRMS: Calcd for $\mathrm{C}_{12} \mathrm{H}_{21} \mathrm{NO}_{9} \mathrm{SNa}[\mathrm{M}+\mathrm{Na}]^{+}$ 378.0829, Found 378.0840.

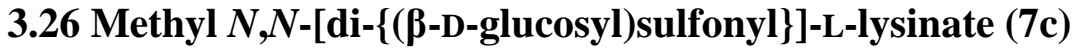


The title compound 7c was obtained from the per-acetylated derivative $\mathbf{7 b}$ following the general procedure 3. Lyophilization afforded the title compound $\mathbf{7 c}$ as a hygroscopic orange solid (90\%): $[\alpha]_{\mathrm{D}}{ }^{25}-22$ (c 1.0, MeOH); $\mathrm{R}_{f} 0.23\left(85: 15 \mathrm{CH}_{3} \mathrm{CN} / \mathrm{H}_{2} \mathrm{O}\right) ;{ }^{1} \mathrm{H}$ NMR (500 MHz, DMSO- $\left.d_{6}\right): \delta 7.60$

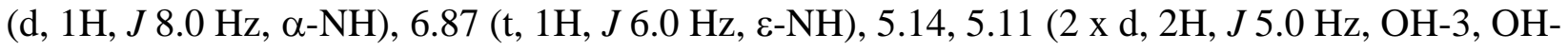
3'), 5.10, 5.05 (2 x d, 2H, J $\left.5.0 \mathrm{~Hz}, \mathrm{OH}-2, \mathrm{OH}-2^{\prime}\right), 5.04$ (m, 2H, OH-4, OH-4'), 4.48, 4.45 (2 x t, 2H, J 6.5 Hz, OH-6, OH-6’), 4.18 (2 x d, 2H, J 9.0 Hz, H-1, H-1'), 3.99 (q, 1H, J 7.0 Hz, $\alpha-\mathrm{CH}$ ), 3.69, 3.63 (2 x dd, 2H, J 7.0, 12.0 Hz, H-6a, H-6a'), 3.66 (s, 3H, OCH $)$, 3.46-3.41 (m, 4H, H-2, H-2', H-6b, H-6b'), 3.27-3.20 (m, H-3, H-3’' H-5, H-5'), 3.10-3.05 (m, 2H, H-4, H-4'), 2.96 (m, $\left.2 \mathrm{H}, \varepsilon-\mathrm{CH}_{2}\right), 1.61\left(\mathrm{~m}, 2 \mathrm{H}, \beta-\mathrm{CH}_{2}\right), 1.43\left(\mathrm{~m}, 2 \mathrm{H}, \delta-\mathrm{CH}_{2}\right), 1.32\left(\mathrm{~m}, 2 \mathrm{H}, \gamma-\mathrm{CH}_{2}\right) ;{ }^{13} \mathrm{C}$ NMR $(125$ MHz, DMSO-d $\left.d_{6}\right): \delta 172.5\left(\mathrm{CO}_{2} \mathrm{CH}_{3}\right), 90.1,88.9(2 \times \mathrm{C}-1), 81.0,80.9(2 \times \mathrm{C}-5), 77.5,77.3(2 \times$ C-3), $70.5(2 \mathrm{C})(2 \times \mathrm{C}-2), 69.7,69.4(2 \times \mathrm{C}-4), 61.2,60.8(2 \times \mathrm{C}-6), 55.9(\alpha-\mathrm{CH}), 51.9\left(\mathrm{OCH}_{3}\right)$, $43.0\left(\varepsilon-\mathrm{CH}_{2}\right), 32.0\left(\beta-\mathrm{CH}_{2}\right), 29.5\left(\delta-\mathrm{CH}_{2}\right), 22.1\left(\gamma-\mathrm{CH}_{2}\right)$; LRMS $\left(\mathrm{ESI}^{+}\right): \mathrm{m} / z 635[\mathrm{M}+\mathrm{Na}]^{+}$; HRMS: Calcd for $\mathrm{C}_{19} \mathrm{H}_{36} \mathrm{~N}_{2} \mathrm{O}_{16} \mathrm{SNa}[\mathrm{M}+\mathrm{Na}]^{+}$635.1398, Found 635.1406.

\subsection{7 $N$-[( $\beta$-D-Glucosyl)sulfonyl]-glycine (2d)}

Per-acetylated glycoconjugate $\mathbf{2 b}(38.3 \mathrm{mg}, 0.08 \mathrm{mmol})$ was added to a solution of a sodium hydroxide in $\mathrm{MeOH}(0.5 \mathrm{M}, 4 \mathrm{~mL})$. The reaction was stirred for $1 \mathrm{~h}$ at $\mathrm{rt}$ then neutralized with Amberlite IR120 $-\mathrm{H}^{+}$resin, filtered and the solvent removed. The remaining residue was dissolved in water and lyophilized. The title compound $\mathbf{2 d}(23.7 \mathrm{mg})$ was obtained as a hygroscopic slightly yellow solid in quantitative yield: $[\alpha]_{\mathrm{D}}{ }^{25}-15$ (c 1.0, MeOH); ${ }^{1} \mathrm{H}$ NMR (500 MHz, DMSO-d 6 ): $\delta 4.23$ (d, 1H, J 9.5 Hz, H-1), 3.67 (d, 2H, J 8.0 Hz, NHCH$), 3.65$ (m, 1H, H6a), 3.43 (dd, 1H, J 6.0, 12.0 Hz, H-6b), 3.42 (t, 1H, J 9.0 Hz, H-2), 3.22 (br t, 2H, J 9.0 Hz, H-3, $\mathrm{H}-5), 3.06$ (t, $1 \mathrm{H}, J 9.0 \mathrm{~Hz}, \mathrm{H}-4) ;{ }^{13} \mathrm{C}$ NMR (125 MHz, DMSO-d $): \delta 171.3\left(\mathrm{CO}_{2} \mathrm{CH}_{3}\right), 90.2(\mathrm{C}-$ 1), 81.4 (C-5), 77.6 (C-3), 70.7 (C-4), 69.7 (C-2), $61.2(\mathrm{C}-6), 44.9\left(\mathrm{NHCH}_{2}\right)$; LRMS (ESI $): m / z$ $324[\mathrm{M}+\mathrm{Na}]^{+}$; HRMS: Calcd for $\mathrm{C}_{8} \mathrm{H}_{15} \mathrm{NO}_{9} \mathrm{SNa}[\mathrm{M}+\mathrm{Na}]^{+}$324.0360, Found 324.0363.

\section{Conclusions}

In summary, we have developed a practical and novel strategy for the conjugation of a glycan moiety to the $\alpha$-amino group of amino acids and to the $\varepsilon$-amino group of lysine. This method provides sulfonamide-linked glycoconjugates from readily available starting materials, the reactions are facile and stereoselective, while the sulfonamide link is stable to both acidic and 
basic reaction conditions. These attributes, together with the already wide use of the sulfonamide group in medicinal chemistry may see considerable utility of these building blocks for the incorporation of sugars into peptides or proteins, pre- or post- peptide synthesis, potentially leading to a powerful additional synthetic tool for chemical biology.

\section{Supplementary Data}

${ }^{1} \mathrm{H}$ NMR and ${ }^{13} \mathrm{C}$ NMR spectra for compounds $\mathbf{2 b - 9 b}, \mathbf{2 c - 7 c}, \mathbf{4} \mathbf{c}^{\prime}, \mathbf{5} \mathbf{c}^{\prime}, \mathbf{2 d}$.

\section{Acknowledgements}

The Australian Research Council is gratefully acknowledged for financial support (Grant No. DP110100071 to S.-A.P.).

\section{References}

1. Gamblin, D. P.; Scanlan, E. M.; Davis, B. G. Chem. Rev. 2009, 109, 131-163.

2. Ernst, B.; Magnani, J. Nat. Rev. Drug Discov. 2009, 8, 661-677.

3. Dove, A. Nat. Biotechnol. 2001, 19, 913-917.

4. Lopez, M.; Drillaud, N.; Bornaghi, L. F.; Poulsen, S.-A. J. Org. Chem. 2009, 74, 2811-2816.

5. Lopez, M.; Bornaghi, L. F.; Innocenti, A.; Vullo, D.; Charman, S. A.; Supuran, C. T.; Poulsen, S.-A. J. Med. Chem. 2010, 53, 2913-2926.

6. Czifrák, K.; Somsák, L. Carbohydr. Res. 2009, 344, 269-277.

7. Zemplén, G. Ber. Deutsch. Chem. Ges. 1926, 59, 1254-1266.

8. Staněk, J.; Černá, J. Tetrahedron Lett. 1963, 4, 35-37.

9. Davis, B. G. Chem. Rev. 2002, 579-601.

10. Hotha, S.; Kashyap, S. J. Org. Chem. 2005, 71, 364-367.

11. Lee, D. J.; Mandal, K.; Harris, P. W. R.; Brimble, M. A.; Kent, S. B. H. Org. Lett. 2009, 11, $5270-5273$.

12. Hang, H. C.; Bertozzi, C. R. Acc. Chem. Res. 2001, 34, 727-736.

13. Moree, W. J.; van der Marel, G. A.; Liskamp, R. J. J. Org. Chem. 1995, 60, 5157-5169.

14. Ibatullin, F. M.; Shabalin, K. A.; Jänis, J. V.; Shavvac, A. G. Tetrahedron Lett. 2003, 44, 7961-7964. 
